\title{
Effect of strain rate on the mechanical properties of a gum metal with various microstructures
}

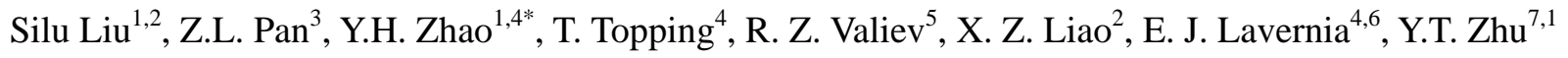 \\ and Q. $\mathrm{Wei}^{3 *}$
}

1. Nano Structural Materials Center, School of Materials Science and Engineering, Nanjing University of Science and Technology, Nanjing 210094, People's Republic of China.

2. School of Aerospace, Mechanical and Mechatronic Engineering, The University of Sydney, NSW 2006, Australia.

3. Department of Mechanical Engineering, University of North Carolina at Charlotte, NC 28223-0001, USA.

4. Department of Chemical Engineering and Materials Science, University of California, Davis, CA 95616, USA.

5. Institute of Physics of Advanced Materials, Ufa State Aviation Technical University, Ufa 450000, Russian Federation.

6. Department of Chemical Engineering and Materials Science, University of California, Irvine, CA 92697, USA.

7. Department of Materials Science and Engineering, North Carolina State University, NC 27695, USA.

* Corresponding authors:

Email: qwei@uncc.edu,yhzhao@njust.edu.cn, Tel./Fax: 86-025-84315304 


\begin{abstract}
In this work, a bulk gum metal (GM) was fabricated via arc melting from high purity powders. The ingots were first extruded using a conventional route followed by equal channel angular pressing (ECAP). The mechanical behavior of the extruded GM and ECAP-processed GM was studied under both quasistatic and high strain rate compression conditions to evaluate the influence of strain rate. In addition, the associated mechanical anisotropy, or the lack thereof, was investigated through loading in different orientations with respect to the extrusion or ECAP direction. Precipitous stress drops were observed under dynamic compression of both extruded and ECAP-processed GM specimens when loading perpendicular to the extrusion direction. Adiabatic shear banding (ASB) was found to be associated with the precipitous stress drops on the dynamic stress-strain curves. The details of the ASBs were characterized by optical and scanning electron microscopy, with emphasis on electron backscattered diffraction (EBSD). The mechanisms responsible for the formation of ASB were examined both from thermal softening and geometrical softening perspectives. Significant microstructure refinement within ASBs was established, and a possible grain refinement mechanism was proposed.
\end{abstract}

Keywords: Gum Metal; ECAP; Adiabatic shear banding; EBSD; Texture; Grain refinement. 


\section{Introduction}

Gum metals (GMs) are essentially a group of $\beta$-Ti alloys containing significant amounts of $\beta$ stabilizers to maintain the body-centered cubic (bcc) lattice structure. GMs have been designed by $a b$ initio calculations to fulfill the requirements of three electronic "magic" numbers so that multiple "super" mechanical properties might be obtained [1-3]. Alloy compositions [1-16], such as Ti-24Nb-4Zr-8Sn-O, Ti-12Ta-9Nb-3V-6Zr-O and Ti-23Nb-0.7Ta-2Zr-O, have been identified to meet those criteria of electronic magic numbers. Additionally, substantial cold work and the presence of certain amount of oxygen have also been found to be indispensable for the reported "super" mechanical properties such as super strength, super elasticity with relatively low elastic modulus, super plasticity and so on $[1,2,12,15,17]$. The combination of these properties might be attributed to the formation of $\mathrm{Zr}$-O clusters, which act as pinning centers that strongly inhibit the movement of dislocations during plastic deformation, giving rise to strong strain hardening [1,3]. Undoubtedly, combined with the bio-compatibility of titanium and its alloys, GMs have been regarded as a promising candidate for biomedical engineering applications, for example, as orthopedic implants as their elastic modulus can be tailored to match that of human bones $[4,5,18,19]$.

As a matter of fact, pure Ti and Ti-alloys have been widely utilized as biomedical materials, such as artificial knee and hip-joints. Among them, Ti-6Al-4V (Ti64) is currently the most commonly used for load bearing applications due to its light weight, high strength, corrosion resistance and relatively low Young's modulus compared to either stainless steels or Co-Cr alloys [20-22]. However, this commercial biomaterial can slowly leach out $\mathrm{V}$ ions which will cause a series of symptoms [4]. Conversely, the transition elements such as $\mathrm{Nb}$, Ta and $\mathrm{Zr}$, etc., are non-toxic and non-allergic alloying elements. Amongst them $\mathrm{Nb}$ and $\mathrm{Ta}$ are $\beta$-stabilizers while $\mathrm{Zr}$ is more or less neutral. Their presence within a GM may render the GM better biocompatibility [4,5]. For example, the relatively low Young's modulus of GMs makes it much more suitable for dental and orthopedic applications. The Young's modulus of Ti64 with $(\alpha+\beta)$ twophase microstructure is $\sim 110 \mathrm{GPa}$, roughly four times larger than that of cortical bone (20-30 GPa). Significant mismatch in Young's modulus between artificial biomedical alloys and cortical bones creates stress shielding effect resulting in low bone density, loosening of implants, implant failure, and an increased likelihood for revision surgery. With less stress shielding, GMs with single $\beta$ phase is attracting significant attention because of their low Young's modulus ranging between $55 \mathrm{GPa}$ and $90 \mathrm{GPa}$. The extremely low Young's modulus ( $\sim 33 \mathrm{GPa})$, quite near that of real human bone, has been reported by Y.L. Hao and co-authors who investigated the elastic deformation of Ti-24Nb-4Zr-8Sn-O [5]. As such, GMs are expected to be potential biomedical materials because of their light weight, high mechanical strength, good biocompatibility and low Young's modulus.

On the basis of first-principles calculations as well as experimental data, alloying can affect the 
stability of $\beta$ phase in GMs. Alloying can also be used to tailor the elastic modulus of GMs along with thermo-mechanical treatment [5,6,18]. Thereby, a combinatory methodology of theoretical studies [6-8] and experimental efforts $[5,11,14-16]$ renders it possible to fabricate $\beta$-phase GMs with desirable properties. When a compositional average valence electron number (electron/atom (e/a)) of ca. 4.24, which turns out to be one of the three electronic magic numbers, is reached, the shear moduli $\mathrm{G}_{011}$ and $\mathrm{G}_{111}$ have the tendency to vanish. $\mathrm{G}_{011}$ corresponds to shearing along $<011>$ on $\{011\}$, and $\mathrm{G}_{111}$ corresponds to shear along <111> on $\{011\},\{112\}$ and $\{123\}$. The ideal shear strength for shearing in a $<111>$ direction on a $\{112\}$ plane is given by the equation $\tau_{\mathrm{m}}=0.11 \mathrm{G}_{111}[3,6,8]$, where $\tau_{\mathrm{m}}$ is the ideal shear strength along all potential shear directions. As such, the ideal shear strength scales linearly with the shear moduli. The nearly vanishing or exceedingly small shear moduli points to relatively low ideal shear strength along those directions when e/a reaches 4.24. In addition, the resolved shear stress (RSS) required to move dislocation may exceed the ideal shear strength of GMs if the pinning obstacles distribute with appropriate spacing. In this sense, the conventional dislocation-mediated plasticity may be entirely inoperative, and GMs may fail at a very high stress level considering the competition of dislocation mobility and ideal shear [1,6,17].

In light of the above, a dislocation-free plastic deformation mechanism has been proposed for GMs. For example, GMs have been observed to deform by giant faults which are macroscopic planar defects with large plastic strain [1]. The generation and multiplication of giant faults are supposed to be closely related to the formation of nano-disturbances [7,8], viz. nanometer scale planar areas of local shear. From the perspective of physical model, abnormal nano-disturbances are described as nanoscale dipoles of nonconventional partial dislocations with arbitrary, non-quantized Burgers vector magnitudes [7]. This mechanism, however, immediately became a subject of intense debate in the community, particularly because of the lack of convincing evidence. Later on, conventional dislocation slipping [9,12,13], twinning $[10,11]$ and phase transformations [11,14-16] have been discovered, which have been employed to explain the peculiar mechanical behavior of GMs.

Notwithstanding the ambiguous picture regarding the plastic deformation mechanism, GMs are drawing increasing attention from researchers concerning elastic deformation, nonlinear elasticity, conventional tensile behavior, in vitro biocompatibility and so on. However, to the best of our knowledge, an important aspect of the mechanical behavior of GMs is missing from the literature: the high strain rate or dynamic mechanical properties [23-26] has largely been ignored. High strain rate behavior is of paramount importance in a number of applications, including implants where varying loading rate in service may well be anticipated. Under impact loading, materials are subjected to large deformation within a very short time frame, and experience a strain rate in the order of $10^{3} \mathrm{~s}^{-1}$, or even higher. Dynamic loading of visco-plastic materials usually brings forth much more complicated processes and 
phenomena vis-à-vis quasi-static loading ( loading usually renders insufficient time for the heat generated from plastic work to be dissipated out of the specimen, and thus adiabatic heating may become an important issue. Localized thermal softening may eventually lead to rapid local plastic deformation and final catastrophic failure of the specimen [2326].

In this work, titanium-based $\beta$-phase GMs with various microstructures were produced by arc melting and casting followed by conventional extrusion and then equal-channel angular press (ECAP). ECAP is among the most popular top-down technologies for the production of ultrafine grain (UFG, grain size $d$ ranging from $\sim 100 \mathrm{~nm}$ to $\sim 1000 \mathrm{~nm}$ ) and even nanocrystalline (NC, $d$ below $\sim 100 \mathrm{~nm}$ ) [27,28]. Mechanical properties of the GM under both quasi-static compression and high-strain rate compression were investigated in order to evaluate the effect of loading rate on the mechanical behavior of GMs at large. Furthermore, loading was in different directions, i.e., extrusion and transverse directions so as to examine the anisotropy of the GM after thermo-mechanical treatment. Optical microscope and scanning electron microscope (SEM) were used to reveal the microstructure of the as-processed materials as well as the microstructure after mechanical loading. In-depth information from electron backscattered diffraction (EBSD) was obtained to assist the interpretation of the formation of adiabatic shear band in the GM under uni-axial compression.

\section{Experimental procedures}

The initial ingot, which was 2.0 inches in diameter and 6.6 inches in length, was made of high purity $\mathrm{Ti}, \mathrm{Nb}$, Ta and $\mathrm{Zr}$ powder through arc melting and casting. Table 1 lists nominal composition of the GM. The microstructure and composition of the ingot are usually homogeneous using this technique. To avoid contamination, a protective layer of Ti film was placed around the powder preform. As such the work piece was encapsulated during arc melting. A billet with a diameter of 0.79 inch was obtained via extruding the ingot at $1010^{\circ} \mathrm{C}$. Subsequently, one pass of ECAP was employed to impose severe plastic deformation to the extruded billet at $500{ }^{\circ} \mathrm{C}$ without back pressure. The ECAP facility employs a stationary constraint die with channel diameter of 0.79 inch and channel angle of 120 degrees. Henceforth the ECAP-processed GM will be designated as ECAP-GM, and the extruded GM as Extruded-GM. Figs. 1(a) and (b) schematically illustrate the fabrication process. It will become clear later that the extrusion direction is of great importance in determining the mechanical properties of the GM specimens. The relative directions are delineated in the sample coordinate system as provided in Fig. 1(c) with reference to the fabrication process.

Two groups of samples with respective dimensions of $(3 \times 3 \times 6 \mathrm{~mm})$ and $(3 \times 3 \times 3 \mathrm{~mm})$ were cut from the Extruded-GM and ECAP-GM materials using wire electrode discharge machining (EDM). The former group is for quasi-static uni-axial compression, while the latter group for high-strain rate uni-axial 
compression. An MTS servohydraulic loading system (strain rates $10^{-4}-10^{0} \mathrm{~s}^{-1}$ ) was used to measure the quasi-static behavior under uniaxial compression. This load frame is equipped with a self-alignment feature, and lubricant was applied between the loading faces of the specimens and the platens to mitigate barreling effect during mechanical loading. The strain rate was monitored through the cross-head speed and machine compliance was not corrected. As such, we did not attempt to derive the elastic constants from the quasi-static compression.

High strain rate uni-axial loading was performed using a Kolsky bar system (or Split Hopkinson Pressure Bar (SHPB) system). In this experiment, the specimen is sandwiched between two co-axial isodiameter elastic bars, called the input and output bars, usually made of very high strength steel (in this work, the elastic bars are made of 350 grade maraging steel). During the experiment, a projectile of the same diameter as and coaxial with the input bar is launched from a gas gun. The projectile impacts the end of the input bar, generating a stress wave which travels down the input bar. At the interface between the specimen and the input bar, part of the wave is reflected as a tensile wave, part of it taken by the specimen, yet another part transmitted to the output bar. Strain gages are attached to the input/output bars and the signals are collected by a high-speed multi-channel oscilloscope. The signals can then be processed to obtain stress-strain data of the specimen. For details of the working principles, applications as well as the cautions and caveats of the Kolsky bar technique, see references [29-33].

To examine the anisotropic behavior, or the lack thereof, loading was applied either in the extrusion direction or in the transverse direction, see Figure 1 (c) for details. Planes XOY and XOZ were polished to a mirror finish so as to examine the plastic deformation in different loading directions upon completion of the mechanical loading.

Post-mortem microstructure observations of the polished surfaces were performed via optical microscope, SEM and EBSD. For this purpose, the recovered samples were mechanically grinded by Buehler EcoMet 250/AutoMet 250 followed by etching using a diluted solution of $1 \mathrm{HF}: 2.5 \mathrm{HNO}_{3}: 100$ $\mathrm{H}_{2} \mathrm{O}$. The etched samples were inspected by an optical microscope - Olympus BX60M. Afterwards, some specimens were further examined by light grinding to remove the etched surface and then electro-polished using an electrolyte of $90 \mathrm{vol}$ \% acetic acid and $10 \mathrm{vol}$. \% perchloric acid at $45 \mathrm{~V}$ at room temperature in Buehler electromet-4. Zeiss Ultra equipped with Oxford Instruments Aztec 2.0 EBSD system was used to further characterize the microstructure of the electro-polished samples. The commercial software Channel 5 was used to optimize the EBSD data, including removal of non-indexed and mis-indexed pixels, and to further explore the orientation information behind the original data. Transfer of texture descriptions from Euler angles to Miller indices is conveniently operated by the aid of an open-source software, Textools authored by University of Montreal, Canada.

Phase constitutions were determined by $2 \theta / \theta$ coupling method of X-ray diffraction (XRD) using a $\mathrm{Cu}$ 
K $\alpha$ irradiation on Rigaku Diffractometer.

\section{Experimental results}

For simplicity, samples obtained through the quasi-static compression of the initial Extruded-GM, with loading direction perpendicular (transverse) or parallel to the extrusion direction, are designated as Extruded-GM-QS1 or Extruded-GM-QS2, respectively; specimens of dynamic loading of the initial Extruded-GM, loaded perpendicular or parallel to the extrusion direction, are designated as ExtrudedGM-DY1 or Extruded-GM-DY2, respectively; quasi-static samples of the initial ECAP-GM loaded perpendicular or parallel to the extrusion direction are denoted as ECAP-GM-QS1 or ECAP-GM-QS2, respectively; dynamic specimens of the initial ECAP-GM loaded perpendicular or parallel to the extrusion direction are denoted as ECAP-GM-DY1 or ECAP-GM-DY2, respectively.

\subsection{Mechanical Properties}

\subsubsection{Quasi-static Compression}

Figs. 2(a) and (b) are the true stress-strain curves of Extruded-GM-QS1 and Extruded-GM-QS2, showing yield strength around $750 \mathrm{MPa}$ along with substantial strain hardening. No dramatic differences of mechanical properties can be identified between these two conditions, suggesting more or less isotropic behavior.

Figs. 2(c) and (d) present the true stress-strain curves of ECAP-GM-QS1 and ECAP-GM-QS2, respectively. The yield strength of the material has been increased to ca. $950 \mathrm{MPa}$. But in both loading directions, the material shows elastic-nearly perfectly plastic behavior - the strain hardening has been greatly diminished upon ECAP processing. Such changes in quasi-static behavior imposed from the ECAP process will bring forth quite significant change in the material's mechanical properties under dynamic loading, as will be shown in the next section.

\subsubsection{High-strain rate behavior}

Dynamic (high-strain rate) mechanical behavior of the extruded material in the two loading directions is presented in Figs. 2(e) and (f). The strain rates are also given in the inset. The peak flow stresses in both loading directions are roughly the same, on the order of $\sim 1200 \mathrm{MPa}$, indicating significant rate dependence of this material. However, for the transverse samples (Fig. 2 (e)), after the peak stress, a precipitous stress drop is recorded in the stress-strain curve, indicating strong tendency to plastic instability under uniaxial dynamic compression, presumably via the occurrence of adiabatic shear localization as will be verified with post-loading examination later in this work. When loaded at high rate in the extrusion direction (Fig. 2 (f)), the extruded specimens exhibit steady flow softening, without 
abrupt stress collapse even to $\sim 40 \%$ total strain.

Figs. $2(\mathrm{~g})$ and $(\mathrm{h})$ show that ECAP processing raises the peak stress of the material under dynamic loading to above $1250 \mathrm{MPa}$. However, in both loading directions, precipitous stress drop occurs. Yet, there is indeed some difference between the two loading conditions: the transverse samples show stress drop almost immediately after they enter into plastic regime, while those loaded in the extrusion direction exhibit some finite uniform plastic deformation before the stress drop takes place, more or less in line with the quasi-static behavior.

\subsection{Microstructure}

\subsubsection{Microstructure of the as-processed material}

Figs. 3(a), (c), (d) and (f) are optical micrographs from XOZ and XOY planes (see Fig. 1) of the Extruded-GM and ECAP-GM samples, respectively. Marble-like structures caused by plastic deformation are observed in XOZ plane after extrusion and ECAP (Figs. 3(a, d)). Figs. 3(b) and (e) are enlarged images from areas of Fig. 3(a) and (d), respectively. Some amount of equiaxed grains around $50 \mu \mathrm{m}$ or less in size are observed. Fig. 3(c) also shows recrystallized grains with the size of a few tens micrometers coexisting with the elongated deformed bands tens to hundreds micrometers wide. This is likely to be a consequence from the high temperature extrusion as well as the shear stress. The ECAP-GM is more severely deformed because of the presence of intensively deformed bands (Fig. 3(f)).

Fig. 4(a) and (b) present areas from XOY plane of Extruded-GM and ECAP-GM, respectively. The $\mathrm{X}$-axis, or the transverse direction, is horizontal in these images. The Y-axis, or the extrusion direction, is vertical. The black lines represent high angle grain boundaries (HAGB, misorientation $\theta>10^{\circ}$ ); the silver lines represent low angle grain boundaries (LAGB, $2^{\circ}<\theta<10^{\circ}$ ). The color code used for grain orientation in all orientation maps is IPF coloring-Z inserted at the lower left corner of Fig. 4(a). From Fig. 4(a), the width of the elongated grains of Extruded-GM is around $150 \mu \mathrm{m}$. A large number of recrystallized equiaxed grains are well developed through dynamic recrystallization by the combined effect of high temperature and plastic deformation. However, the widths of elongated grains of ECAP-GM are around $100 \mu \mathrm{m}$ as estimated from Fig. 4(b). Densely distributed LAGB can also be observed compared to Fig. 4(a). Cells inside the elongated grains have evolved through the accumulation and interaction of defects. As reported before [27,28], grain refinement and the development of defect structure take place during ECAP. The texture information from EBSD data will be discussed in the next part and analyzed to interpret the formation of adiabatic shear band.

\subsubsection{Quasi-static post-loading microstructure examinations}

The post-loading microstructure corresponding to the XOZ plane of the Extruded-GM-QS1 has been 
observed by optical microscope and a representative image is shown in Fig. 5(a). Compared with the microstructure of Extruded-GM (Fig. 3(a)), swirled band-like deformation structures are observed to be distributed inside the marble-like pattern in Fig. 5(a) and so is the wide range of grain size from several microns to tens of microns. According to [9], marble-like deformation structure may be considered as the curly grains or swirled structure which have also been found in tungsten, iron, niobium and other body centered cubic (bcc) metal rods highly deformed either by rotary swaging or by wire drawing. Such marble-like structure can thus serve as evidence that grains of the quasi-statically loaded Extruded-GMQS1 have been further deformed and stress distribution becomes increasingly inhomogeneous compared to the Extruded-GM. More specifically, some grains have been fragmented into small ones with a broad grain size distribution. The XOY plane of the quasi-statically compressed Extruded-GM-QS2 is also inspected by optical microscopy with a representative image displayed in Fig. 5(b), showing almost the same elongated deformed strips as well as recrystallized grains with size of tens of microns, as depicted in Fig. 3(c).

Marble-like structure from XOZ plane of quasi-statically compressed ECAP-GM-QS1 is shown in Fig. 5(c), which is similar to the as-ECAP counterpart (Fig. 3(d)). This, however, does not mean that no microstructure modification has taken place during the quasi-static compression of the ECAP-GM. For instance, apparent microgrooves aggregate to form chunks or bands of plastic flow in the marble-like pattern of ECAP-GM-QS1. Some drastic changes can be identified when comparison is made between the morphologies of the XOY plane of ECAP-GM-QS2 and ECAP-GM. For example, Fig. 5(d) displays intensive shear bands cutting into the elongated deformation bands, forming a wavy structure in the XOY plane of ECAP-GM-QS2. The wavy structure of the shear band might indicate grain rotation and twisting under quasi-static compression. Since plastic flow is localized in the shear band, this shearing structure can be regarded as consistent with the elastic-nearly perfect plastic stress-strain curves where strain hardening has been nearly diminished upon ECAP (Fig. 2 (f)).

\subsubsection{Dynamic post-loading microstructure examinations}

It has been noticed that under uniaxial dynamic compression, the stress-strain curves of the extruded GM exhibit precipitous stress drop immediately upon yielding (Fig. 2 (e)). Post-loading surface morphology examination shows the occurrence of adiabatic shear banding (ASB) in XOZ plane of the Extruded-GM-DY1 specimen, Figs. 6(a), (b) and (c). The loading direction is vertical in these figures. Fig. 6(a) provides the SEM image of the whole specimen recovered from the high strain rate uniaxial compression. Multiple ASBs and cracks can be clearly identified. The primary ASBs form conjugated patterns. An optical micrograph of the conjugated ASBs from Fig. 6(a) is displayed in Fig. 6(b). Two shear bands start from the top left part and top right part, propagate through the diagonal path, and 
conjugate at the lower middle part of the sample. The ASBs are roughly at $45^{\circ}$ with respect to the loading direction, corresponding to the maximum shear stress plane. Wavy plastic flow lines run into the ASB and curve away from the other side of the ASB, forming a so-called canonical structure of ASB in the sense of Wright [34]. Filamentary structures can be distinguished in Fig. 6(c) which is the enlarged optical micrograph of the left branch of the ASBs in Fig. 6(b). These filamentary structures might be the giant faults reported in the tensile experiment of GMs [1].

Figs. 6(d), (e) and (f) show post-loading surface morphology of the XOY plane of Extruded-GMDY2. The loading direction is vertical in these figures. When loaded in this direction, the extruded GM exhibits apparently uniform deformation and no strong evidence of plastic instability can be identified from the post-loading surface examinations, in keeping with the high-strain rate stress-strain curve shown in Fig. 2. At higher magnification (Fig. 6(e)), surface steps, presumably from the kinking process [5,12], can be observed. Fig. 6(f) is the optical micrograph of Extruded-GM-DY2. Elongated deformed bands and strips can be observed in this image with some recrystallized equiaxed grains. However, the primary microstructure features are still from the extrusion process.

Post-dynamic loading surface morphologies of the ECAP-GM samples loaded in two different directions are presented in Fig. 7. Figs. 7(a), (b) and (c) characterize post-loading surface in the XOZ plane (ECAP-GM-DY1). The loading direction is vertical in these images. Adiabatic shear bands and cracks can be clearly observed from the SEM image, similar to Fig. 6 (a) for the extruded GM after dynamic loading. Again, both primary and secondary ASBs are observed and the primary ASBs are conjugated. All ASBs are at $\sim 45^{\circ}$ with respect to the loading axis. In other words, they form along the maximum shear stress plane. Higher magnification images of the ASBs (Figs. 7 (b) and (c)) of this sample reveal the canonical ASB pattern in the sense of Wright [34].

Post-dynamic loading surface morphology in the XOY plane of ECAP-GM-DY2 is presented in Fig. 7(d), (e) and (f) where the loading direction is vertical. Adiabatic shear bands that have not fully developed can be identified from the SEM image in the corners of the specimen (Fig. 7(d)), presumably due to the limited strain level as suggested by the dynamic stress-strain curves for this kind of specimens (Fig. 2). However, higher magnification images of Figs. 7 (e) and (f) still show substantial localized shear flow along with equiaxed grains from dynamic recrystallization. Here elongated deformed strips and bands are swirled compared with Fig. 3(f) which is the view of XOY plane of the ECAP-GM. Fig. 7(f) provides a relatively distinct view of those recrystallized grains. In this figure, the ribbons of recrystallized grains were largely deformed to have a wavy appearance.

Finally, Fig. 8(a) and (b) are inverse pole figure (IPF)-Z orientation maps from EBSD of the XOZ planes of Extruded-GM-DY1 and ECAP-GM-DY1, respectively. From these two maps, a striking observation can be made regarding the microstructure within the ASB, in the vicinity of the boundary 
between the ASB and the matrix, as well as the matrix with much coarser grains. Widths of two shear bands are both near $\sim 30 \mu \mathrm{m}$. First of all, remarkable grain refinement has occurred within the ASB, resulting in ultrafine equiaxed grains. This suggests great temperature rise within the ASB during dynamic loading. Evidence of shear flow can be identified of the grains within the ASB near the $\mathrm{ASB} /$ matrix boundaries, indicating the dynamic process and temperature and strain gradients across the ASB regions. Finally, the canonical ASB pattern is clearly revealed by these IPF orientation maps.

\section{$3.3 \mathrm{XRD}$}

Fig. 9 is the XRD results of Extruded-GM and ECAP-GM. In Fig. 9(a), a single (110) peak was identified in Extruded-GM, which implies single $\beta$ phase and strong texture (fiber texture is in accordance with EBSD). In ECAP-GM, as indicated by Fig. 9(b), new $\alpha$ "-martensite was discovered to emerge from the $\beta$-phase and the original fiber texture has been randomized.

\section{Discussion}

In this part, in-depth discussion will be provided regarding the experimental results observed and presented in the previous section. It will be focused on the effect of thermomechanical processing on the microstructure and mechanical behavior of the GM, and the factors responsible for the formation of ASB in the material under dynamic loading.

\subsection{Effect of extrusion and ECAP on the microstructure and mechanical behavior of the Gum metal}

The as-cast ingot of the $\beta$-alloy is composed of nearly $100 \%$ bcc $\beta$-phase, as confirmed by $x$-ray diffraction. This is not surprising as the primary alloying elements are beta-stabilizers ( $\mathrm{Nb}$ and $\mathrm{Ta}$, see Table 1). However, evidence for the appearance of $\alpha$ "-martensite has been detected in the thermomechanically processed material, as shown by the XRD results in Fig. 9. Fig. 9 (a) is the XRD results from the Extruded-GM, which show strong texture with only $\beta$ (110) peak identified. This is consistent with extrusion texture of bcc metals and alloys [35]. Fig. 9 (b) shows the XRD results of ECAP-GM. First of all, it is observed that ECAP has greatly randomized the texture of the metal. Furthermore, weak peaks of $\alpha$ "-martensite phase can be identified. This is in accordance with literature results on $\beta$-titanium alloys with similar composition where rolling can induce martensitic transformation [36-38]. Results reported in [39] seem in contrast to ours. However, we need to notice that temperature and strain conditions were different in our experiment and these parameters are critical to phase transition. As such, under considerable shear stress such as experienced during ECAP, stress-induced martensitic transformation can take place. Because the peaks from the $\alpha^{\prime \prime}$ phase are all quite weak, we refrain from quantifying its content using XRD. 
In the previous section, it has been noticed that ECAP has greatly increased the strength of the Gum metal. While grain size refinement from ECAP could definitely be a factor contributing to the strength increase, it should not be surprising that the precipitation of $\alpha^{\prime \prime}$ particles should also serve to strengthen the $\beta$-alloy. As such, the increase in quasi-static peak stress from $\sim 750 \mathrm{MPa}$ of the Extruded-GM to $\sim 925$ MPa of the ECAP-GM can be a consequence of the combined effect of grain size refinement and the appearing of the $\alpha^{\prime \prime}$-martensite. That being said, a quantitative estimation as to which mechanism contributes how much to the strength increase is beyond the scope of this work. But based on the observation that the ECAP-GM exhibits largely diminished strain hardening, it is believed that grain size refinement might be the primary mechanism for strength increase. In other words, the volume fraction of the martensite phase might be still too low to participate in strain hardening as observed in some specially treated Al-alloys [40].

Overall, the mechanical properties of the GM specimens under quasi-static loading are consistent with the results of other ultrafine grained bcc metals such as tungsten, iron, tantalum, etc. [29$31,33,41,42]$, and face-centered cubic metals such as copper and nickel $[42,43]$. In those UFG metals, strain hardening also appears to decrease with decreasing grain size, esp. in the UFG/NC regime. It has been proposed that there is an intrinsic size effect on work hardening in metals [44,45]. The size effect may be interpreted by the reduced slip length and dislocation interaction with reduced grain size, or source starvation in the case of single crystals $[42,46,47]$. For UFG/NC metals the grain size becomes so small that dislocation accumulation inside grains becomes difficult due to the interaction of dislocations with grain boundaries [48]. Under tension [46], strain hardening helps to delay or even suppress plastic instability (necking) in the sense of Considere's criterion (it should be noted that the Considere's criterion is for rate-independent materials) [49]. Under compression, it mitigates the tendency to shear localization, particularly at high strain rate where adiabatic temperature rise within the specimen due to insufficient time for the heat generated from plastic work to be dissipated out of the specimen [23-26]. In the same line of argument, ECAP-GM with refined grain size is unable to maintain sufficient strain hardening to sustain stable and uniform plastic deformation. The nearly flat true stress-strain curve of ECAP-GM-QS1 (Fig. 2(e)), suggests the increased tendency of the transition of plastic deformation from stable and uniform mode to unstable and inhomogeneous mode. Such tendency becomes even stronger from Extruded-GM-QS2 to ECAP-GM-QS2 (compare Fig. 2(b) and Fig. 2(f)). Moreover, the transition of plastic deformation mode due to different strain hardening capacity can also be found from the microstructure characterizations. Shear bands can be seen in Fig. 5(d) for ECAP-GM-QS2, indicating unstable and inhomogeneous plastic deformation.

4.2. Effect of loading rates on the mechanical behavior 
The plastic behaviors as shown in Figs. 2(e) and Fig. 2(g) point to catastrophic failure of ExtrudedGM-DY1 and ECAP-GM-DY1 samples caused by flow softening. During high strain rate plastic deformation, a large quantity of the plastic work is converted into heat [25]. Due to the very short time frame available for the heat dissipation into the environment, adiabatic temperature rise becomes a significant issue as it causes thermal softening and even severe plastic localization within the specimen. This may in turn lead to catastrophic failure of the specimen [23-26]. The intense localized shearing is commonly called adiabatic shear banding (ASB). While the global adiabatic temperature rise of the whole specimen can be only a few tens of degrees, the adiabatic temperature rise inside an ASB can be as high as $>1000^{\circ} \mathrm{C}$, depending on the loading condition and materials parameters. For a first-order estimation, the temperature rise within an ASB under dynamic loading can be calculated as

$$
\theta=\beta \frac{\tau \gamma}{\rho c}
$$

where $\theta$ is the adiabatic temperature rise in the ASB, $\beta$ the Taylor-Quinney factor representing the adiabaticity of the process, usually taken to be 0.9 for high strain rate loading such as used in this work, $\tau$, $\gamma$ the shear stress and shear strain associated with the ASB, $\rho$ the density and $c$ is the specific heat capacity of the specimen. To use Eqn. (1), $\tau$ can be taken as the flow stress of the specimen using a Taylor factor $\mathrm{M}$ of $3.0[\tau=\sigma / 3$, where $\sigma$ is the flow stress of the specimen to be read from Fig. 2], $\gamma$ can be measured from Fig. $8(\mathrm{a}, \mathrm{b})$, which is tangent of the angle between the ASB boundary and the curved flow line. For example, the angle is about $\sim 30^{\circ}$ as measured for ECAP-GM-DY from Figure 8 (b), which is translated to a shear strain of $\tan 30^{\circ}=0.577$. Since the predominant element of the GM is $\mathrm{Ti}$, it is believed to be safe to use the value of Ti for $c\left(25.0 \mathrm{~J} / \mathrm{K} . \mathrm{mol}\right.$.) and $\rho\left(5.5 \mathrm{~g} / \mathrm{cm}^{3}\right)$ (see table 1). According to Equation (1), the adiabatic temperature rise within the ASB might be estimated as high as $1510{ }^{\circ} \mathrm{C}$ for Extruded-GM-DY1 and $1570{ }^{\circ} \mathrm{C}$ for ECAP-GM-DY1. It should be noted that this estimate is a conservative one since the shear strain experienced in the center of the ASB must be much higher than the boundary. It should also be obvious that there is a temperature gradient across the ASB. Such adiabatic temperature rise within the ASB should be sufficiently high to render dynamic recrystallization, which leads to the much refined grain size within the ASB seen in Figure 8.

Under quasi-static loading, such ASB is absent as evidenced both from the stress strain plots presented in Fig. 2 and the post-mortem surface examinations. As such, it is believed that strain rate should have a strong effect on the occurrence, or the lack thereof, of ASB in this Ti-based GM. That being said, the ultimate tendency to ASB is a combined effect of many factors, as explored in Refs. [23-26]. More details regarding the formation of ASB will be addressed in a later section. Roughly speaking, factors affecting ASB tendency can be viewed into four categories: (i) the stress state; (ii) loading conditions such as temperature and strain rate; (iii) intrinsic factors such as the microstructure of the 
material (precipitations, inclusions, voids, etc.); (iv) intrinsic physical properties such as density, heat capacity, etc.

\subsection{Geometric/Texture Softening and the formation of ASB}

The mechanical properties and the corresponding microstructures suggest that Extruded-GM-DY1 and Extruded-GM-DY2, as well as ECAP-GM-DY1 and ECAP-GM-DY2, exhibit dramatic differences that call for further explanation. Flow softening is identified in Extruded-GM-DY2 and ECAP-GM-DY2. Post-mortem SEM of Extruded-GM-DY2 and ECAP-GM-DY2 reveals ASB. Stress drops are found in the stress-strain curves of Extruded-GM-DY1 and ECAP-GM-DY1. Furthermore, both SEM and optical microscope show ASB and cracks. That is to say, adiabatic localized shearing is exacerbated in ExtrudedGM-DY1 and ECAP-GM-DY1. Why the difference? Here it is believed the different behaviors stem from the geometric relationship between the loading direction and the extrusion direction. In other words, the difference is caused by the anisotropy induced by thermomechanical processing. Such anisotropic mechanical behavior is associated with the texture developed in the bcc GM. Considering the two loading directions, plane XOY of Extruded-GM and ECAP-GM were further studied by orientation information from EBSD.

It has been noticed that visco-plastic materials with high flow stress, high thermal sensitivity, low strain hardening and low strain rate sensitivity (SRS) exhibit increased susceptibility to ASB. As such, results presented in Refs. [23-26,29-33] are consistent with the present results. However, we believe the difference in mechanical behavior, particularly the occurrence of ASB or the lack thereof, is rooted in the texture in the specimens of this work.

Fig. 4(a) characterizes an arbitrary area from the XOY plane (Fig. 1) of Extruded-GM by EBSD. It has been proposed that swaging can induce $\langle 110\rangle$ fibrous texture in bcc metals [35]. Since swaging is similar to extruding in terms of stress condition, the fibrous texture is consistent with the present result. The inverse pole figure (IPF)-Y given in Fig. 10 (a), of the Extruded-GM shows that $\langle 110\rangle$ fibrous texture is parallel to $\mathrm{Y}$, the extrusion direction. Furthermore, Euler angles are utilized to reveal the texture components more clearly. Taking into consideration the sample geometry and crystal symmetry [50], two sections $\left(\varphi_{2}=0^{\circ}\right.$ and $\left.\varphi_{2}=45^{\circ}, 0^{\circ}<\varphi_{1}, \psi<90^{\circ}\right)$ selected from the Euler space or orientation distribution function (ODF) are adopted to measure the dominant texture components in Extruded-GM. For instance, Euler angles $\left(\varphi_{1}, \psi, \varphi_{2}\right)$ read from its ODF represents the specific orientation or unequivocal orientation. Among them, magnitude of $\varphi_{1}$ and $\psi$ can be read from axis $\varphi_{1}, \psi$ of the ODF, $\varphi_{2}$ is $0^{\circ}$ or $45^{\circ}$. The ODF is provided in Fig. 10 (b). Judging from such results, the dominant texture is $\left(90^{\circ}, 20^{\circ}, 45^{\circ}\right)$ with the strongest texture intensity. The Euler angles $\left(90^{\circ}, 20^{\circ}, 45^{\circ}\right)$ are equivalent to the Miller indices (114) $[\overline{2} 21]$. Crystallographic plane (114) of the crystals is parallel to the rolling plane, while the 
crystallographic direction $[\overline{2} \overline{2} 1]$ points to the rolling direction. According to the default setting of Fig. 1, the normal to the rolling plane is parallel to the normal direction (axis Z) of the sample coordinate system, and the rolling direction is defined as the transverse direction (axis X). As such, crystallographic directions [ $\overline{2} 21],[1 \overline{1} 0]$ and [114] are corresponding to the axis $\mathrm{X}, \mathrm{Y}$ and $\mathrm{Z}$ of the sample coordinate system, respectively. Its orientation has been illustrated by the 3D crystal model in Fig. 10 (c).

An arbitrary area on XOY plane of ECAP-GM was selected for EBSD. Its orientation image is given in Fig. 4(b). Since ECAP might trigger many texture components [51-53], it is inappropriate to attempt to pin down the dominant texture component by pole-figure (PF) or IPF and Euler angles would still be adopted to analyze the texture components. Fig. 10 (d) displays the two selected Euler space sections from its ODF. The criteria of selection are the same as the stated Euler space sections of the Extruded-GM. Fig. 10 (d) shows that $\left(70^{\circ}, 40^{\circ}, 0^{\circ}\right)$ is the dominant texture component as viewed from the texture intensity. The Euler angles $\left(70^{\circ}, 40^{\circ}, 0^{\circ}\right)$ is equivalent to the Miller indices (011) [1 $\left.\overline{2} 2\right]$. That is,

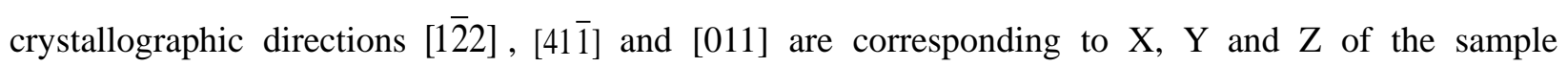
coordinate system, respectively. Fig. 10 (e) represents this orientation by 3D crystal view.

In order to interpret the adiabatic shear localization, the average Schmid factor is calculated based on the texture information derived above [54,55]. Schmid factor $m$ is defined as: $m=\cos \lambda \cos \Phi$, where $\lambda$ and $\Phi$ are the angles between the normal stress and the normal to the slip plane and slip direction, respectively. Soft directions with high magnitude of Schmid factor could thus be sorted out. The loading direction is in the transverse direction $(\mathrm{X})$ for the "perpendicular" geometry; otherwise, it is in the extrusion direction (Y) for the "parallel" geometry. As a consequence, the intrinsic weak directions with the lowest critical shear stress correspond to $<011>$ on plane $\{011\},<111>$ on planes $\{011\},\{112\}$ and $\{123\}[5,6,8]$. These orientations should be susceptible to shearing. So far, all the components in the analysis of Schmid factor have been confirmed.

In the case of Extruded-GM-DY1, the X-axis of as-Extruded-GM is loaded under dynamic impact condition from the view of sample coordinate system, so $[\overline{2} 21]$ is the loading stress direction. On the other hand, in the case of Extruded-GM-DY2, the Y-axis of Extruded-GM is loaded under dynamic condition, and $[1 \overline{1} 0]$ is the loading stress direction. Utilizing these two loading directions with those shear directions (intrinsically susceptible to shear), Schmid factors of Extruded-GM have been calculated and listed in Table 2 .

When it comes to the case of ECAP-GM-DY1, the X-axis of the ECAP-GM is loaded under dynamic condition which is $[1 \overline{2} 2]$. On the other hand, in the case of ECAP-GM-DY2, the Y-axis of 
ECAP-GM is loaded under dynamic condition, and so [41 $\overline{1}]$ is the loading direction for Schmid factor analysis. Utilizing these two loading directions with those shear directions (intrinsically susceptible to shear), Schmid factors of the ECAP-GM have been calculated and listed in Table 3.

It should be noticed here that $\lambda$ and $\Phi$ should be taken between $0^{\circ}$ and $180^{\circ}$ so that the Schmid factor values are in the range from -0.5 to 0.5 . Negative Schmid factors means compressive stress while positive Schmid factors are for tensile stress. It has also been proposed that the absolute value of Schmid factor should be larger than 0.3 to practically activate the shearing process [54]. Therefore shear directions with Schmid factors below 0.3 are not considered in this work. Based on these results, it is not hard to discover that shear directions for these cases, Extruded-GM-DY1 and ECAP-GM-DY1, are primarily [111] and [11] $]$, respectively. However, the calculated Schmid factors in both cases of Extruded-GM-DY2 and ECAP-GM-DY2 indicate that different shear directions have been activated. So when the sample is dynamically loaded in the "perpendicular" geometry, the activated shear direction would be in the primarily favorable orientation. This should exacerbate adiabatic shear localization compared to different shear directions so that ASBs and even crack have formed. Such texture/geometric softening that eventually results in ASB has been observed in other bcc metals such as W [56].

\subsection{Microstructural Evolution of ASB}

The Schmid factor can also shed light on the formation and propagation of ASB with the assistance of EBSD data [54,55]. As shown in Fig. 8(a), the image from Extruded-GM-DY1 can be divided into the matrix (Ma), and ASB region (Sa). ODF of area Ma is displayed in Fig. 11(a). From this Euler angles $\left(50^{\circ}\right.$, $\left.35^{\circ}, 0\right)$ which are equivalent to Miller indices (023) [3 $\left.\overline{3} 2\right]$ represent the dominant texture component of the matrix. Fig. 11(b) is the ODF of area Sa. Euler angles $\left(44^{\circ}, 40^{\circ}, 0\right)$ and $\left(50^{\circ}, 90^{\circ}, 45^{\circ}\right)$, equivalent to (011) [3 $2 \overline{2}]$ and (110) [1 $\overline{1} 2]$, respectively, are relatively strong texture components in the ASB. Similarly, ECAP-GM-DY1 in Fig. 8(b) can also be separated into the matrix (Mb), and ASB region (Sb). Fig. 11(c) and (d) provide the ODF results of areas $\mathrm{Mb}$ and $\mathrm{Sb}$. These ODF figures show strong texture component indicated by Euler angles $\left(25^{\circ}, 90^{\circ}, 45^{\circ}\right)$ for the matrix of ECAP-GM-DY1, and weak and multiple texture components indicated by Euler angles $\left(6^{\circ}, 40^{\circ}, 0\right)$ and $\left(45^{\circ}, 40^{\circ}, 0\right)$ for the ASB of ECAP-GM-DY 1. These Euler angles correspond to Miller indices (110)[3 $\overline{3} 2],(011)[100]$ and (110) [3 $\overline{2} 2]$ via Textools. It might be ascribed to the recrystallization within the ASB such that diverse texture components formed and emerged in the process. The interaction between dynamic recrystallization and newly developed texture components renders the texture intensity largely reduced. [3 $\overline{3} 2]$ is taken into the calculation of Schmid factors for the matrix of both Extruded-GM-DY1 and ECAP-GM-DY1. The result is given in 
Table 4. Based on this, the activated shear direction is again [ $\overline{1} 11]$ in the matrix.

The shear stress component imposed on the samples should be responsible for the orientation evolution. Though changed, the orientation of the matrix can still induce single-direction orientated shearing according to the results of Table 4. As has been found out through orientation analysis given above, the ASBs are composed of diverse and relatively weak texture components. This should largely be due to the dynamic recrystallization (DRx) caused by the significant adiabatic temperature rise within the ASB $[24,25]$. In reality, the DRx process, when combined with the extremely short time-frame, also provides an explanation for the observed UFG microstructure within the ASB (Figs. 8 (a,b)).

In the development of ASB, localized shear will no longer be orientated in a single predominant direction and DRx will eventually lead to the formation of ultrafine grains with more or less randomized orientations. This grain refinement process is schematically illustrated in Fig. 12. Regions A, B and C in Fig. 12 (a) represent typical conditions in this experiment. Regions A and B are the areas affected by localized adiabatic heating under dynamic loading. Moreover, region A is sheared in a single predominant direction. However, region $\mathrm{B}$ is sheared in varying directions, which would smear out the strong texture developed before. Region $\mathrm{C}$ indicates the uniform plastic deformation independent of loading geometry under quasi-static condition. In the absence of localized adiabatic heating and the subsequent DRx, plasticity in this region is supposed to be stable and homogeneous. In other words, region A will become increasingly unstable and may eventually lead to shear failure. It is noticed that Region A represents plastic deformation under dynamic loading with "perpendicular" loading geometry. During the formation of ASB, lattice rotation may occur according to the plastic deformation of continuum medium. Fig. 13 displays rotation of two potential orientations in the stereo-projection. As shown in Figs. 12 (b) and (c), the coarse grains might be fragmented by the shear stress components of multi-direction to form much refined grains. The combining effect of multi-direction shearing and DRx substantially refined those grains inside ASBs to $\sim 500 \mathrm{~nm}$ in Extruded-GM-DY1 and ECAP-GM-DY1. Besides, samples (ExtrudedGM-DY2 and ECAP-GM-DY2) under dynamic loading without evident ASB have varying shear directions activated. Shearing in varying directions may avoid, at least to a certain degree, the occurrence of flow localization. It can thus be concluded that the single predominant shear direction would be conducive to the occurrence of adiabatic shear localization, which would eventually facilitate the generation and propagation of ASB and even crack. On the other hand, varying shear directions may render delocalization and make the sample decreasingly susceptible to adiabatic shear failure. But it may promote grain refinement inside the adiabatic shear band to accommodate more plastic deformation.

It is then believed that the different experimental results from the different samples and loading geometries are a consequence of a number of factors. Traditional wisdom says that "slip" events during mechanical loading will gradually and eventually rotate crystals to align with the loading direction. This 
also reminds us that lattice rotation might have been incurred to accommodate the large strain so that orientations have been remarkable modified [7,8,15,57]. The change in orientation can be verified by the color change in the IPF-Z orientation map. Through further inspection of the matrix in Figs. 8(a) and (b), the color change within individual grains is believed to be caused by lattice rotation. Representative areas are selected to characterize the lattice rotation in Extruded-GM-DY1 and ECAP-GM-DY1, as highlighted by the white rectangular shapes A and B, C and D in Fig. 8(a) and (b), respectively. As the color changes gradually, the corresponding area rotates its orientation. This can be specifically manifested by their PFs and 3D crystal views in Fig. 13. From Fig. 13(a), \{100\} PF of area A and B indicates the orientation change of these two areas through the rotation of their corresponding projected poles. 3D crystal views in Fig. 13(b) and (c) are plotted to characterize this rotation process. $\{100\} \mathrm{PF}$ of area $\mathrm{C}$ and $\mathrm{D}$ are given in Fig. 13(d) and their corresponding 3D crystal views are plotted in Fig. 13(e) and (f). It can also explain the lattice rotation from the isolated matrix area to the adjacent area in the ASB of Fig. 8(b).

\section{Summary and Concluding Remarks}

In this work, a titanium-based gum metal (GM) has been fabricated by casting followed by extrusion and ECAP. Uniaxial quasi-static and dynamic compression were performed in different loading geometries with regard to the work piece to evaluate the mechanical properties of the GM. Based on the experimental results and in-depth discussions, the following conclusions have been reached.

(1) The as-cast and extruded GM shows single beta-phase. Extrusion produces strong texture. ECAP further refines the microstructure and at the same time remarkably smears out the extrusioninduced texture. ECAP also promotes the occurrence of $\beta \rightarrow \alpha$ " martensitic transformation.

(2) Thermomechanical processing via extrusion and ECAP have largely diminished strain hardening of the GM. Hot-extrusion also renders relatively strong anisotropy in mechanical behavior both under quasi-static and dynamic compression.

(3) Plastic deformation mode of Extruded-GM and ECAP-GM under dynamic compression changes from stable and homogeneous mode to unstable and inhomogeneous mode.

(4) Adiabatic shear bands are observed in Extruded-GM-DY1 and ECAP-GM-DY1 and catastrophic stress drops are detected from the stress-strain curves of these samples. ExtrudedGM-DY2 and ECAP-GM-DY2 display thermal softening, though no evident adiabatic shear bands are found in these samples.

(5) Both texture/geometric softening and thermal softening are held responsible for the occurrence of ASB in the Extruded-GM and ECAP-GM samples when loaded in the "perpendicular" geometry. This is supported by detailed Schmid factor calculations based on EBSD data.

(6) Lattice rotations of individual grains occur when the adiabatic shear band is formed. Multi- 
direction shearing along with dynamic recrystallization within the adiabatic shear band contributes to the process of substantial grain refinement.

\section{Acknowledgements}

This work has been supported by the Office of Naval Research (Grant number N00014-08-1-0405). Silu Liu wishes to acknowledge support from the Graduated School of NJUST and the University of Sydney. Q. Wei is supported by US Army Research Laboratory under Contract No. W911NF-14-0061. E. J. Lavernia wishes to acknowledge ARO W911NF-16-1-0269 for support of his research. Y.H. Zhao would like to acknowledge support by the National Natural Science Foundation of China (51225102 and 2012CB932203). X.Z. Liao wishes to acknowledge the Australian Research Council (DP150101121) for support.

\section{References:}

[1] T. Saito, Multifunctional Alloys Obtained via a Dislocation-Free Plastic Deformation Mechanism, Science. 300 (2003) 464-467. doi:10.1126/science.1081957.

[2] J.-P. Liu, Y.-D. Wang, Y.-L. Hao, Y. Wang, Z.-H. Nie, D. Wang, Y. Ren, Z.-P. Lu, J. Wang, H. Wang, X. Hui, N. Lu, M.J. Kim, R. Yang, New intrinsic mechanism on gum-like superelasticity of multifunctional alloys, Sci. Rep. 3 (2013). doi:10.1038/srep02156.

[3] T. Furuta, S. Kuramoto, J.W. Morris, N. Nagasako, E. Withey, D.C. Chrzan, The mechanism of strength and deformation in Gum Metal, Scr. Mater. 68 (2013) 767-772. doi:10.1016/j.scriptamat.2013.01.027.

[4] K.Y. Xie, Y. Wang, Y. Zhao, L. Chang, G. Wang, Z. Chen, Y. Cao, X. Liao, E.J. Lavernia, R.Z. Valiev, B. Sarrafpour, H. Zoellner, S.P. Ringer, Nanocrystalline $\beta$-Ti alloy with high hardness, low Young's modulus and excellent in vitro biocompatibility for biomedical applications, Mater. Sci. Eng. C. 33 (2013) 3530-3536. doi:10.1016/j.msec.2013.04.044.

[5] Y.L. Hao, S.J. Li, S.Y. Sun, C.Y. Zheng, R. Yang, Elastic deformation behaviour of Ti-24Nb-4Zr7.9Sn for biomedical applications, Acta Biomater. 3 (2007) 277-286. doi:10.1016/j.actbio.2006.11.002.

[6] T. Li, J.W. Morris, N. Nagasako, S. Kuramoto, D.C. Chrzan, "Ideal” Engineering Alloys, Phys. Rev. Lett. 98 (2007). doi:10.1103/PhysRevLett.98.105503.

[7] M.Y. Gutkin, T. Ishizaki, S. Kuramoto, I.A. Ovid'ko, N.V. Skiba, Giant faults in deformed Gum Metal, Int. J. Plast. 24 (2008) 1333-1359. doi:10.1016/j.ijplas.2007.09.009.

[8] M.Y. Gutkin, T. Ishizaki, S. Kuramoto, I.A. Ovid'ko, Nanodisturbances in deformed Gum Metal, Acta Mater. 54 (2006) 2489-2499. doi:10.1016/j.actamat.2006.01.027.

[9] H. Xing, J. Sun, Q. Yao, W.Y. Guo, R. Chen, Origin of substantial plastic deformation in Gum Metals, Appl. Phys. Lett. 92 (2008) 151905. doi:10.1063/1.2908040.

[10] H. Tobe, H.Y. Kim, T. Inamura, H. Hosoda, S. Miyazaki, Origin of $\{332\}$ twinning in metastable $\beta$ Ti alloys, Acta Mater. 64 (2014) 345-355. doi:10.1016/j.actamat.2013.10.048.

[11] H. Xing, J. Sun, Mechanical twinning and omega transition by $\langle 111\rangle\{112\}$ shear in a metastable $\beta$ titanium alloy, Appl. Phys. Lett. 93 (2008) 031908. doi:10.1063/1.2959183.

[12] Y. Yang, S.Q. Wu, G.P. Li, Y.L. Li, Y.F. Lu, K. Yang, P. Ge, Evolution of deformation mechanisms of $\mathrm{Ti}-22.4 \mathrm{Nb}-0.73 \mathrm{Ta}-2 \mathrm{Zr}-1.34 \mathrm{O}$ alloy during straining, Acta Mater. 58 (2010) 2778-2787. doi:10.1016/j.actamat.2010.01.015.

[13] P. Castany, M. Besse, T. Gloriant, Dislocation mobility in gum metal $\beta$-titanium alloy studied via in 
situ transmission electron microscopy, Phys. Rev. B. 84 (2011). doi:10.1103/PhysRevB.84.020201.

[14] Y.B. Wang, Y.H. Zhao, Q. Lian, X.Z. Liao, R.Z. Valiev, S.P. Ringer, Y.T. Zhu, E.J. Lavernia, Grain size and reversible beta-to-omega phase transformation in a Ti alloy, Scr. Mater. 63 (2010) 613-616. doi:10.1016/j.scriptamat.2010.05.045.

[15] J.P. Cui, Y.L. Hao, S.J. Li, M.L. Sui, D.X. Li, R. Yang, Reversible Movement of Homogenously Nucleated Dislocations in a $\beta$-Titanium Alloy, Phys. Rev. Lett. 102 (2009). doi:10.1103/PhysRevLett.102.045503.

[16] S. Kuramoto, T. Furuta, J. Hwang, K. Nishino, T. Saito, Elastic properties of Gum Metal, Mater. Sci. Eng. A. 442 (2006) 454-457. doi:10.1016/j.msea.2005.12.089.

[17] Y.L. Hao, S.J. Li, B.B. Sun, M.L. Sui, R. Yang, Ductile Titanium Alloy with Low Poisson's Ratio, Phys. Rev. Lett. 98 (2007). doi:10.1103/PhysRevLett.98.216405.

[18] S.J. Li, R. Yang, M. Niinomi, Y.L. Hao, Y.Y. Cui, Z.X. Guo, Phase transformation during aging and resulting mechanical properties of two Ti-Nb-Ta-Zr alloys, Mater. Sci. Technol. 21 (2005) 678-686. doi:10.1179/174328405X43108.

[19] S.. Li, R. Yang, M. Niinomi, Y.. Hao, Y.. Cui, Formation and growth of calcium phosphate on the surface of oxidized Ti-29Nb-13Ta-4.6Zr alloy, Biomaterials. 25 (2004) 2525-2532. doi:10.1016/j.biomaterials.2003.09.039.

[20] K. Anselme, M. Bigerelle, B. Noel, E. Dufresne, D. Judas, A. Iost, P. Hardouin, Qualitative and quantitative study of human osteoblast adhesion on materials with various surface roughnesses, J. Biomed. Mater. Res. 49 (2000) 155-166. doi:10.1002/(SICI)1097-4636(200002)49:2<155::AIDJBM2>3.0.CO;2-J.

[21] K. Anselme, P. Linez, M. Bigerelle, D. Le Maguer, A. Le Maguer, P. Hardouin, H.F. Hildebrand, A. Iost, J.M. Leroy, The relative influence of the topography and chemistry of TiAl6V4 surfaces on osteoblastic cell behaviour, Biomaterials. 21 (2000) 1567-1577. doi:10.1016/S01429612(00)00042-9.

[22] J. Lausmaa, Surface spectroscopic characterization of titanium implant materials, J. Electron Spectrosc. Relat. Phenom. 81 (1996) 343-361. doi:10.1016/0368-2048(95)02530-8.

[23] S.M. Walley, Shear Localization: A Historical Overview, Metall. Mater. Trans. A. 38 (2007) 26292654. doi:10.1007/s11661-007-9271-x.

[24] R.W. Armstrong, S.M. Walley, High strain rate properties of metals and alloys, Int. Mater. Rev. 53 (2008) 105-128. doi:10.1179/174328008X277795.

[25] B. Dodd, Y. Bai, Y. Bai, eds., Adiabatic shear localization: frontiers and advances, 2nd ed, Elsevier, Amsterdam ; Boston, 2012.

[26] B. Dodd, Adiabatic shear localization occurrence, theories and applications, Elsevier, Oxford, 2012.

[27] R.Z. Valiev, T.G. Langdon, Principles of equal-channel angular pressing as a processing tool for grain refinement, Prog. Mater. Sci. 51 (2006) 881-981. doi:10.1016/j.pmatsci.2006.02.003.

[28] Y.H. Zhao, X.Z. Liao, Z. Jin, R.Z. Valiev, Y.T. Zhu, Microstructures and mechanical properties of ultrafine grained $7075 \mathrm{Al}$ alloy processed by ECAP and their evolutions during annealing, Acta Mater. 52 (2004) 4589-4599. doi:10.1016/j.actamat.2004.06.017.

[29] Q. Wei, T. Jiao, K. Ramesh, E. Ma, L. Kecskes, L. Magness, R. Dowding, V. Kazykhanov, R. Valiev, Mechanical behavior and dynamic failure of high-strength ultrafine grained tungsten under uniaxial compression, Acta Mater. (2005). doi:10.1016/j.actamat.2005.08.031.

[30] S. Cheng, Y. Zhao, Y. Guo, Y. Li, Q. Wei, X.-L. Wang, Y. Ren, P.K. Liaw, H. Choo, E.J. Lavernia, High Plasticity and Substantial Deformation in Nanocrystalline NiFe Alloys Under Dynamic Loading, Adv. Mater. 21 (2009) 5001-5004. doi:10.1002/adma.200901991.

[31] Q. Wei, L. Kecskes, T. Jiao, K.T. Hartwig, K.T. Ramesh, E. Ma, Adiabatic shear banding in ultrafine-grained Fe processed by severe plastic deformation, Acta Mater. 52 (2004) 1859-1869. doi:10.1016/j.actamat.2003.12.025.

[32] Q. Wei, D. Jia, K.T. Ramesh, E. Ma, Evolution and microstructure of shear bands in nanostructured Fe, Appl. Phys. Lett. 81 (2002) 1240-1242. doi:10.1063/1.1501158.

[33] Q. Wei, H. Zhang, B. Schuster, K. Ramesh, R. Valiev, L. Kecskes, R. Dowding, L. Magness, K. Cho, 
Microstructure and mechanical properties of super-strong nanocrystalline tungsten processed by high-pressure torsion, Acta Mater. 54 (2006) 4079-4089. doi:10.1016/j.actamat.2006.05.005.

[34] T.W. Wright, G. Ravichandran, Canonical aspects of adiabatic shear bands, Int. J. Plast. 13 (1997) 309-325. doi:10.1016/S0749-6419(97)80002-2.

[35] W.Y. Guo, H. Xing, J. Sun, X.L. Li, J.S. Wu, R. Chen, Evolution of Microstructure and Texture during Recrystallization of the Cold-Swaged Ti-Nb-Ta-Zr-O Alloy, Metall. Mater. Trans. A. 39 (2008) 672-678. doi:10.1007/s11661-007-9433-x.

[36] H. Matsumoto, S. Watanabe, S. Hanada, Beta TiNbSn Alloys with Low Young\&amp;rsquo;s Modulus and High Strength, Mater. Trans. 46 (2005) 1070-1078. doi:10.2320/matertrans.46.1070.

[37] H. Matsumoto, S. Watanabe, S. Hanada, Microstructures and mechanical properties of metastable $\beta$ TiNbSn alloys cold rolled and heat treated, J. Alloys Compd. 439 (2007) 146-155. doi:10.1016/j.jallcom.2006.08.267.

[38] S. Guo, Q.K. Meng, X.N. Cheng, X.Q. Zhao, Deformation behavior of metastable $\beta$-type Ti-25Nb2Mo-4Sn alloy for biomedical applications, J. Mech. Behav. Biomed. Mater. 38 (2014) 26-32. doi:10.1016/j.jmbbm.2014.06.006.

[39] M. Besse, P. Castany, T. Gloriant, Mechanisms of deformation in gum metal TNTZ-O and TNTZ titanium alloys: A comparative study on the oxygen influence, Acta Mater. 59 (2011) 5982-5988. doi:10.1016/j.actamat.2011.06.006.

[40] Y.-H. Zhao, J.F. Bingert, X.-Z. Liao, B.-Z. Cui, K. Han, A.V. Sergueeva, A.K. Mukherjee, R.Z. Valiev, T.G. Langdon, Y.T. Zhu, Simultaneously Increasing the Ductility and Strength of Ultra-FineGrained Pure Copper, Adv. Mater. 18 (2006) 2949-2953. doi:10.1002/adma.200601472.

[41] Q. Wei, Z.L. Pan, X.L. Wu, B.E. Schuster, L.J. Kecskes, R.Z. Valiev, Microstructure and mechanical properties at different length scales and strain rates of nanocrystalline tantalum produced by highpressure torsion, Acta Mater. 59 (2011) 2423-2436. doi:10.1016/j.actamat.2010.12.042.

[42] Q. Wei, S. Cheng, K.. Ramesh, E. Ma, Effect of nanocrystalline and ultrafine grain sizes on the strain rate sensitivity and activation volume: fcc versus bcc metals, Mater. Sci. Eng. A. 381 (2004) 71-79. doi:10.1016/j.msea.2004.03.064.

[43] J. Su, W.G. Guo, L.J. Kecskes, S.N. Mathaudhu, Q. Wei, A rate dependent constitutive model for ECAE Cu based on instrumented nanoindentation results, Mater. Sci. Eng. A. 597 (2014) 279-287. doi:10.1016/j.msea.2013.12.090.

[44] R.Z. Valiev, Y. Estrin, Z. Horita, T.G. Langdon, M.J. Zehetbauer, Y.T. Zhu, Fundamentals of Superior Properties in Bulk NanoSPD Materials, Mater. Res. Lett. 4 (2016) 1-21. doi:10.1080/21663831.2015.1060543.

[45] Y.T. Zhu, X.Z. Liao, X.L. Wu, Deformation twinning in nanocrystalline materials, Prog. Mater. Sci. 57 (2012) 1-62. doi:10.1016/j.pmatsci.2011.05.001.

[46] D. Jia, Y.M. Wang, K.T. Ramesh, E. Ma, Y.T. Zhu, R.Z. Valiev, Deformation behavior and plastic instabilities of ultrafine-grained titanium, Appl. Phys. Lett. 79 (2001) 611-613. doi:10.1063/1.1384000.

[47] A.W. Thompson, Effect of grain size on work hardening in nickel, Acta Metall. 25 (1977) 83-86. doi:10.1016/0001-6160(77)90249-8.

[48] Y.T. Zhu, X. Liao, Nanostructured metals: Retaining ductility, Nat. Mater. 3 (2004) 351-352. doi:10.1038/nmat1141.

[49] E.. Hart, Theory of the tensile test, Acta Metall. 15 (1967) 351-355. doi:10.1016/00016160(67)90211-8.

[50] O. Engler, V. Randle, Introduction to texture analysis: macrotexture, microtexture, and orientation mapping, 2nd ed, CRC Press, Boca Raton, 2010.

[51] I.J. Beyerlein, L.S. Tóth, Texture evolution in equal-channel angular extrusion, Prog. Mater. Sci. 54 (2009) 427-510. doi:10.1016/j.pmatsci.2009.01.001.

[52] S. Li, I.J. Beyerlein, M.A.M. Bourke, Texture formation during equal channel angular extrusion of fcc and bcc materials: comparison with simple shear, Mater. Sci. Eng. A. 394 (2005) 66-77. doi:10.1016/j.msea.2004.11.032. 
[53] A.A. Gazder, F.D. Torre, C.F. Gu, C.H.J. Davies, E.V. Pereloma, Microstructure and texture evolution of bcc and fcc metals subjected to equal channel angular extrusion, Mater. Sci. Eng. A. 415 (2006) 126-139. doi:10.1016/j.msea.2005.09.065.

[54] E. Bertrand, P. Castany, I. Péron, T. Gloriant, Twinning system selection in a metastable $\beta$-titanium alloy by Schmid factor analysis, Scr. Mater. 64 (2011) 1110-1113. doi:10.1016/j.scriptamat.2011.02.033.

[55] J.H. Shen, Y.L. Li, Q. Wei, Statistic derivation of Taylor factors for polycrystalline metals with application to pure magnesium, Mater. Sci. Eng. A. 582 (2013) 270-275. doi:10.1016/j.msea.2013.06.025.

[56] Q. Wei, L.J. Kecskes, K.T. Ramesh, Effect of low-temperature rolling on the propensity to adiabatic shear banding of commercial purity tungsten, Mater. Sci. Eng. A. 578 (2013) 394-401. doi:10.1016/j.msea.2013.04.109.

[57] J.L. Sun, P.W. Trimby, F.K. Yan, X.Z. Liao, N.R. Tao, J.T. Wang, Shear banding in commercial pure titanium deformed by dynamic compression, Acta Mater. 79 (2014) 47-58. doi:10.1016/j.actamat.2014.07.011. 


\section{Figure captions}

Fig. 1. Schematic sketches of material fabrication process and sample coordinate system. (a) Extrusion process with the green arrow indicating the extrusion direction; (b) ECAP process with sample coordinate system; (c) sample coordinate system employed in (a) and (b) with the red, green, blue arrows indicating transverse direction (TD, axis $\mathrm{X}$ ), extrusion direction (ED, axis $\mathrm{Y}$ ) and normal direction (ND, axis Z), respectively.

Fig. 2. True stress-strain curves of Extruded-GM and ECAP-GM. (a) Extruded-GM-QS1; (b) Extruded-GM-QS2; (c) ECAP-GM-QS1; (d) ECAP-GM-QS2; (e) Extruded-GM-DY1; (f) Extruded-GM-DY2; (g) ECAP-GM-DY1; (h) ECAP-GM-DY2. Note: DY-dynamic; QS-Quasistatic; per-perpendicular to extrusion; par-parallel to extrusion.

Fig. 3. Optical micrographs of Extruded-GM and ECAP-GM. (a) Plane XOZ of Extruded-GM; (b) high magnification to show detailed local features in (a); (c) plane XOY of Extruded-GM; (d) plane XOZ of ECAP-GM; (e) high magnification to show detailed local feature in (d); (f) plane XOY of ECAP-GM.

Fig. 4. (a) Orientation map of plane XOY of Extruded-GM; (b) orientation map of plane XOY of ECAP-GM. The black line represents grain boundary with the misorientation larger than 10 degrees and the silver line represents low angle grain boundary with the misorientation between 2 and 10 degrees. The color code used for grain orientation in all orientation maps is IPF coloring-Z inserted at the lower left corner of Fig. 4(a). They are identical codes in all orientation maps.

Fig. 5. Optical micrographs of (a) plane XOZ of the Extruded-GM-QS1; (b) plane XOY of Extruded-GM-QS2; (c) plane XOZ of ECAP-GM-QS1; (d) plane XOY of ECAP-GM-QS2. The loading direction is horizontal in these graphs.

Fig. 6. Microstructure of Extruded-GM-DY1 and Extruded-GM-DY2. (a) SEM view of plane XOZ of Extruded-GM-DY1; (b) optical micrograph of plane XOZ of Extruded-GM-DY1; (c) high magnification image to show detailed local feature in (b); (d) SEM view of plane XOY of Extruded-GM-DY2; (e) high magnification image to show detailed local feature in (d); (f) optical micrograph of plane XOY of Extruded-GM-DY2. The loading direction is vertical in these graphs.

Fig. 7. Microstructure of ECAP-GM-DY1 and ECAP-GM-DY2. (a) SEM view of plane XOZ of ECAP-GM-DY1; (b) optical micrograph of plane XOZ of ECAP-GM-DY1; (c) high magnification image to show detailed local feature ib (b); (d) SEM view of plane XOY of ECAP-GM-DY2; (e) optical micrograph of plane XOY of ECAP-GM-DY2; (f) high magnification image to show detailed local feature in (e). The loading direction is vertical in these graphs.

Fig. 8. (a) Orientation map of plane XOZ of Extruded-GM-DY1, this map is separated to matrix - Ma and adiabatic shear band - Sa by white arrows; (b) orientation map of plane XOZ of 
ECAP-GM-DY1, this map is also divided to matrix - Mb and adiabatic shear band - Sb by white arrows. The loading direction is horizontal in these graphs.

Fig. 9. (a) XRD result of Extruded-GM showing strong texture and only one peak corresponding to the bcc $\beta$-phase; (b) XRD result of ECAP-GM. Evidence of $\alpha$ "-martensite can be observed and the texture has been reduced.

Fig. 10. (a) IPF-Y of Fig. 4(a); (b) ODF of Fig. 4(a); (c) 3D crystal view of the dominant texture component in Fig. 4(a); (d) ODF of Fig. 4(b); (e) 3D crystal view of the dominant texture component in Fig. 4(b). The corresponding color scale bar indicates the texture intensity in all texture-related maps. The code for the colorful lines in 3D crystal views is indicated in Fig. 1.

Fig. 11. (a) ODF of matrix Ma in Fig. 8(a); (b) ODF of adiabatic shear band Sa in Fig. 8(a); (c) ODF of matrix Mb in Fig. 8(b); (d) ODF of adiabatic shear band Sb in Fig. 8(b).

Fig. 12. Schematic graph of the formation of (a) adiabatic shear band and (b), (c) grain refinement within the band. Regions $\mathrm{A}$ and $\mathrm{B}$ are the areas affected by localized heat in dynamic impact. Moreover, region A is sheared in single unequivocal direction, however, region B is sheared in varying directions. Region $\mathrm{C}$ indicates the deformation under quasi-static compression regardless of the geometric relationship.

Fig. 13. (a) PFs of area A and B highlighted by white rectangular shape in Fig. 8(a); (b) 3D crystal view of the orientation of area A; (c) 3D crystal view of the orientation of area B; (d) PFs of area $\mathrm{C}$ and $\mathrm{D}$ highlighted by white rectangular shape in Fig. 8(b); (e) 3D crystal view of the orientation of area C; (f) 3D crystal view of the orientation of area D.

Table 1: The nominal composition of Ti alloy of this work.

Table 2: Schmid factors of activated shear directions by compressing in TD and ED of ExtrudedGM.

Table 3: Schmid factors of activated shear directions by compressing in TD and ED of ECAPGM.

Table 4: Schmid factors of activated shear directions by compressing in crystallographic direction $[3 \overline{3} 2]$. 


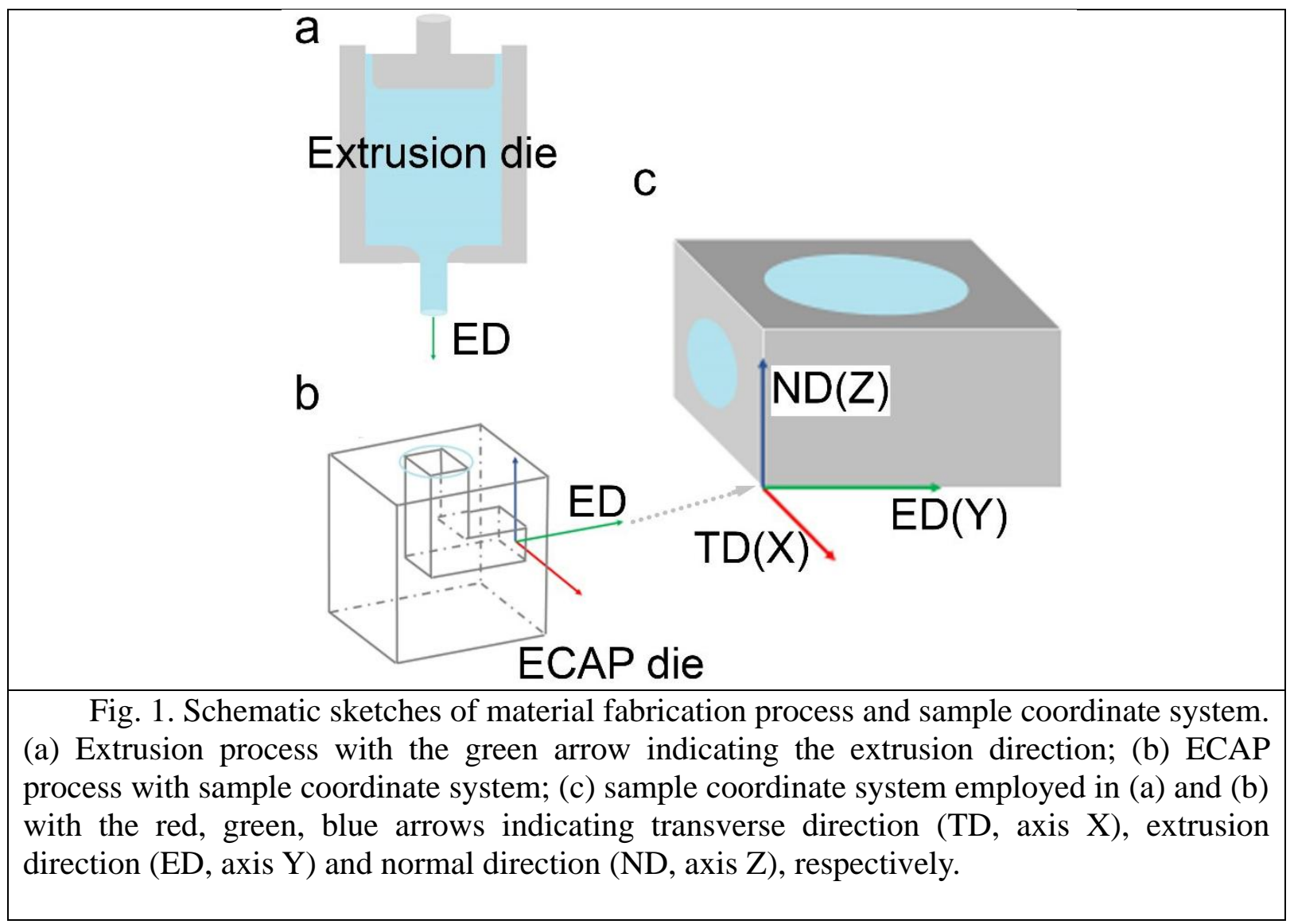




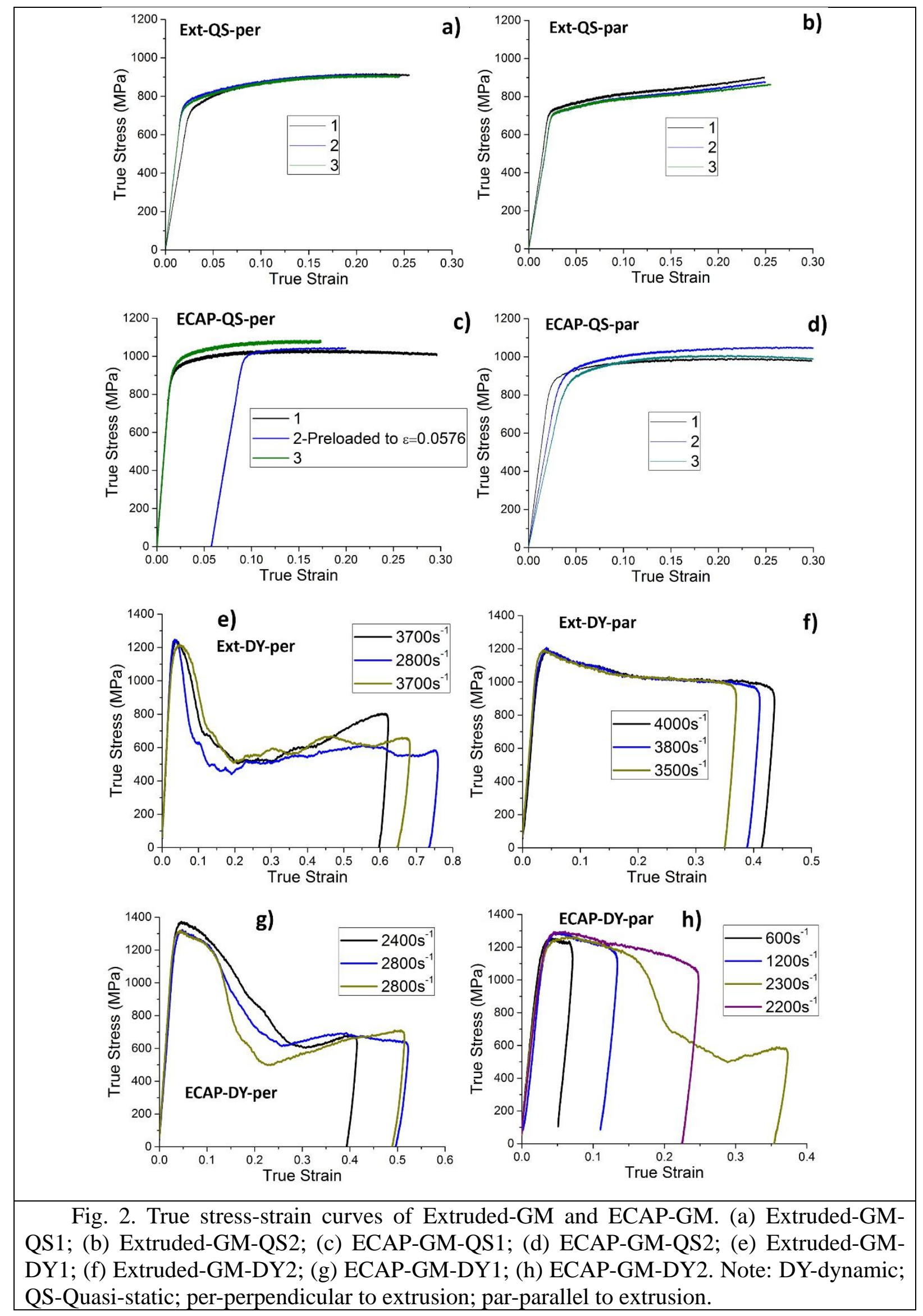




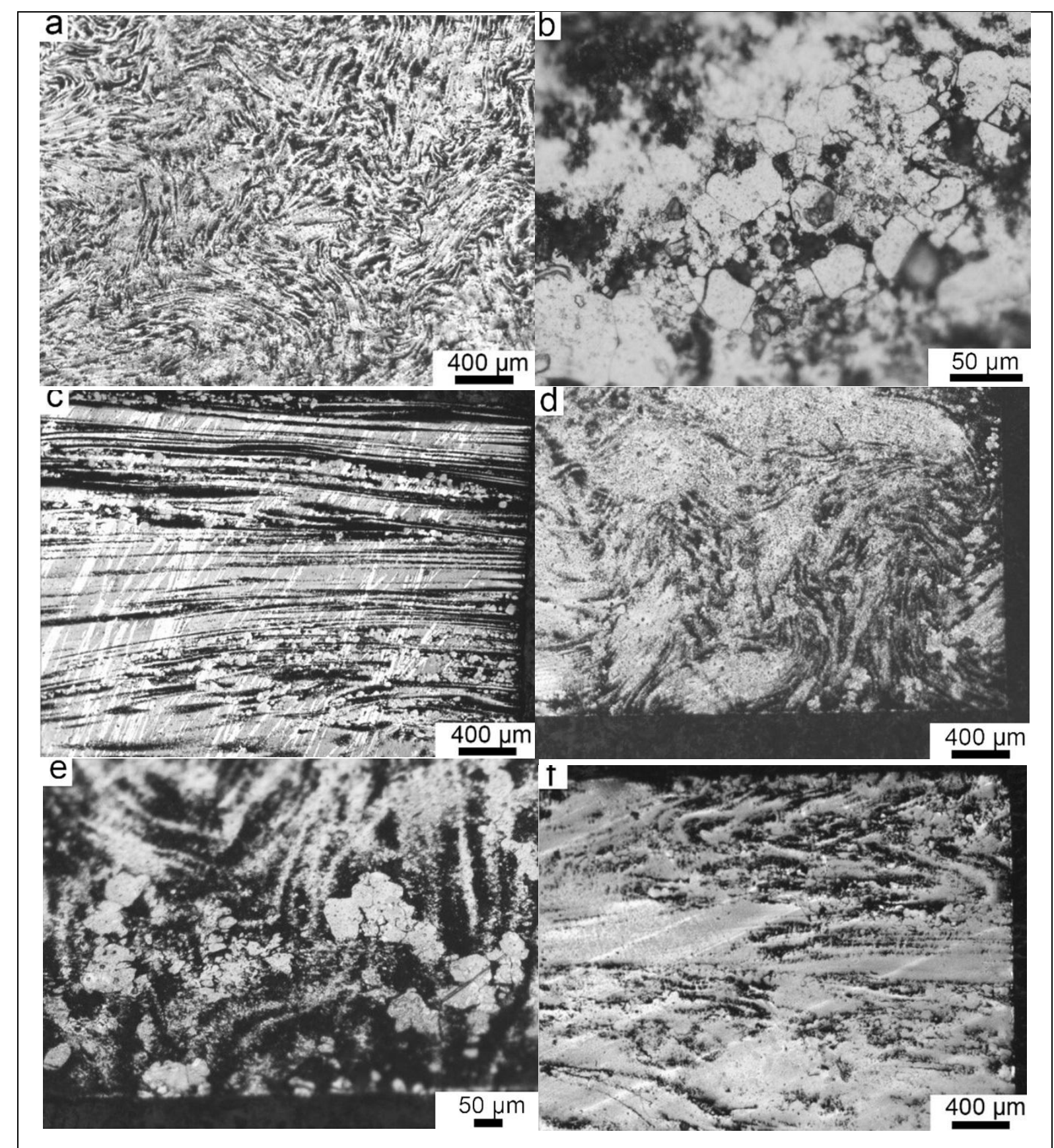

Fig. 3. Optical micrographs of Extruded-GM and ECAP-GM. (a) Plane XOZ of Extruded-GM; (b) high magnification to show detailed local features in (a); (c) plane XOY of Extruded-GM; (d) plane XOZ of ECAP-GM; (e) high magnification to show detailed local feature in (d); (f) plane XOY of ECAP-GM. 


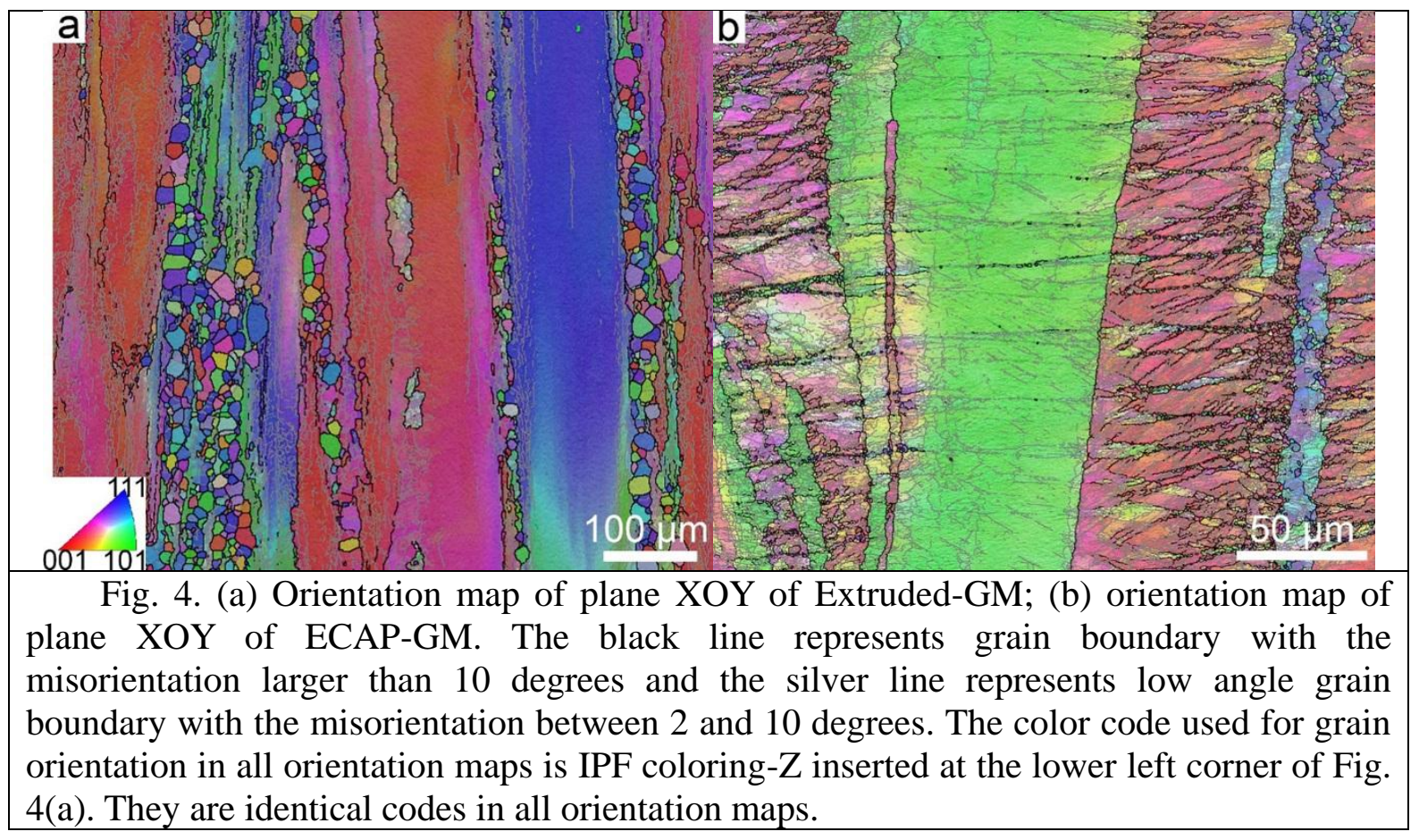




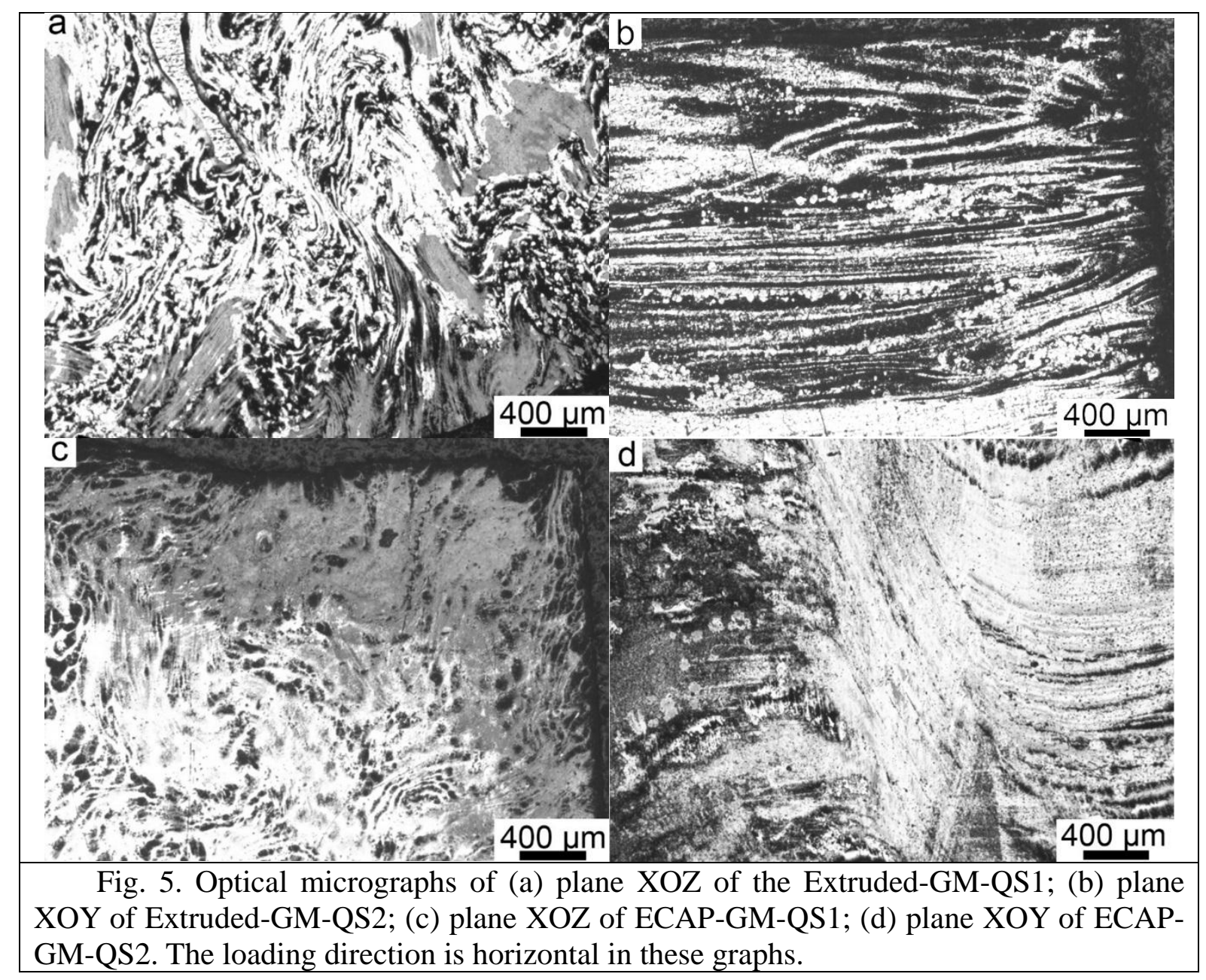




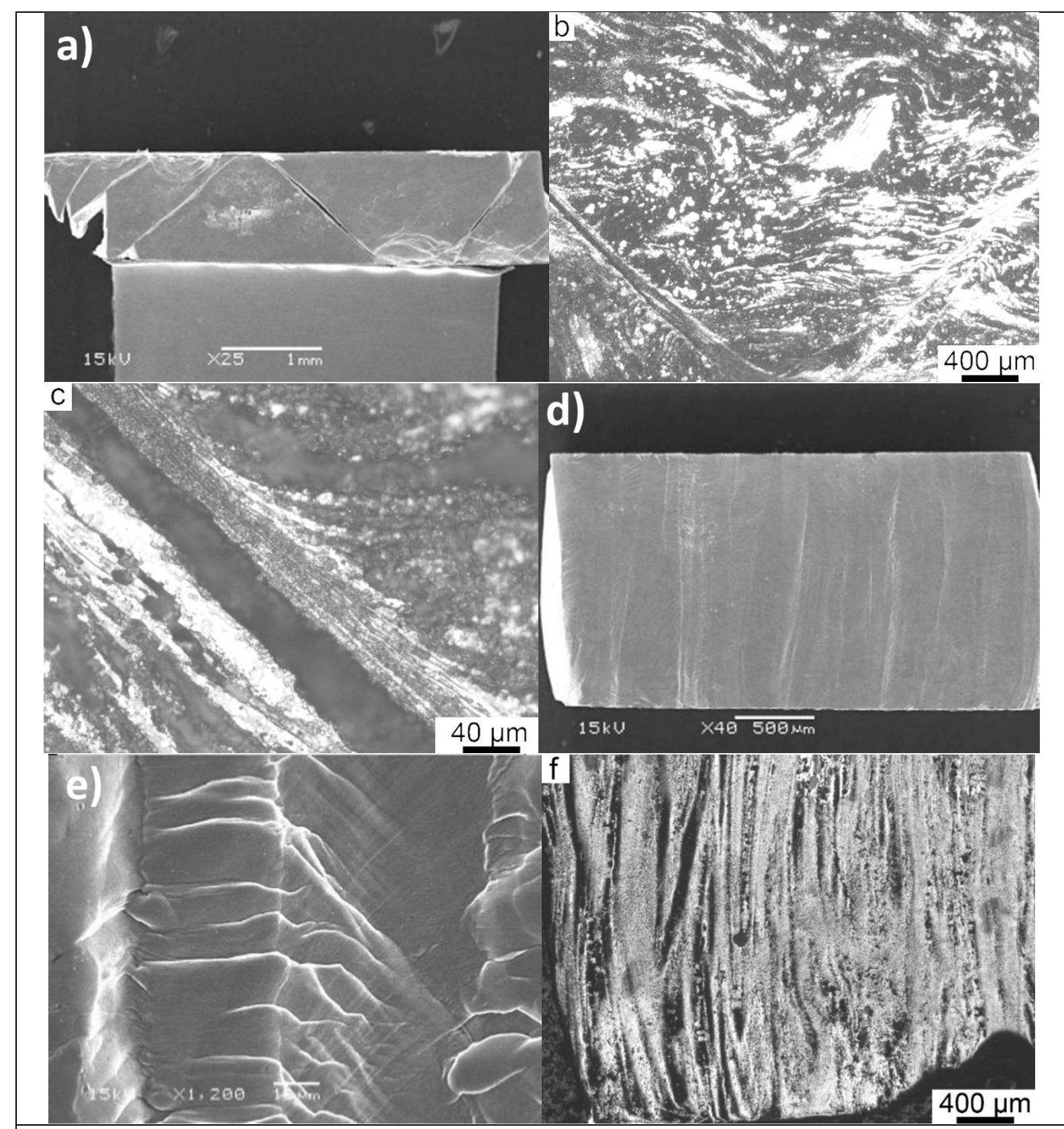

Fig. 6. Microstructure of Extruded-GM-DY1 and Extruded-GM-DY2. (a) SEM view of plane XOZ of Extruded-GM-DY1; (b) optical micrograph of plane XOZ of ExtrudedGM-DY1; (c) high magnification image to show detailed local feature in (b); (d) SEM view of plane XOY of Extruded-GM-DY2; (e) high magnification image to show detailed local feature in (d); (f) optical micrograph of plane XOY of Extruded-GM-DY2. The loading direction is vertical in these graphs. 


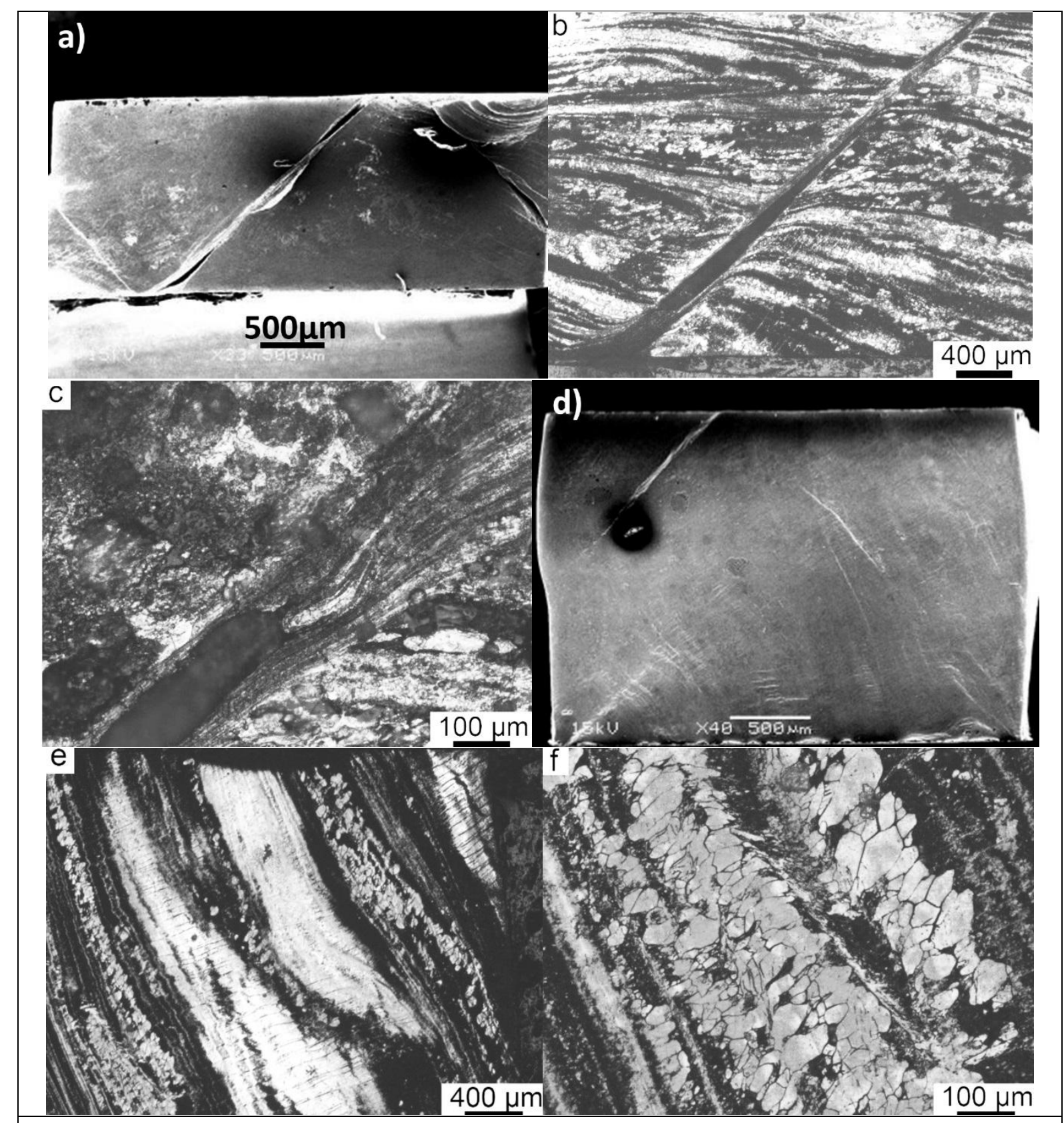

Fig. 7. Microstructure of ECAP-GM-DY1 and ECAP-GM-DY2. (a) SEM view of plane XOZ of ECAP-GM-DY1; (b) optical micrograph of plane XOZ of ECAP-GM-DY1; (c) high magnification image to show detailed local feature ib (b); (d) SEM view of plane XOY of ECAP-GM-DY2; (e) optical micrograph of plane XOY of ECAP-GM-DY2; (f) high magnification image to show detailed local feature in (e). The loading direction is vertical in these graphs. 


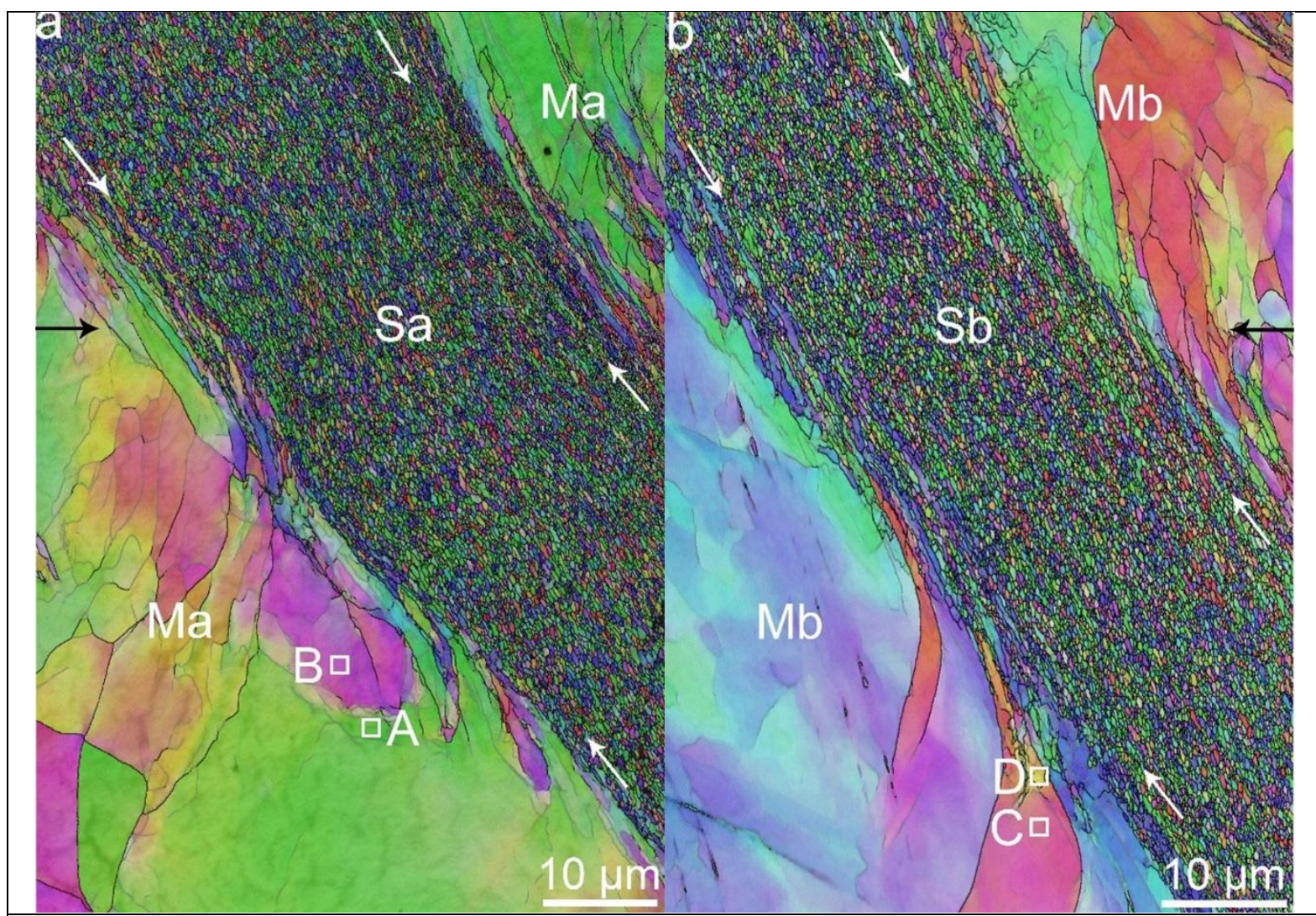

Fig. 8. (a) Orientation map of plane XOZ of Extruded-GM-DY1, this map is separated to matrix - Ma and adiabatic shear band - Sa by white arrows; (b) orientation map of plane XOZ of ECAP-GM-DY1, this map is also divided to matrix - Mb and adiabatic shear band - Sb by white arrows. The loading direction is horizontal in these graphs. 

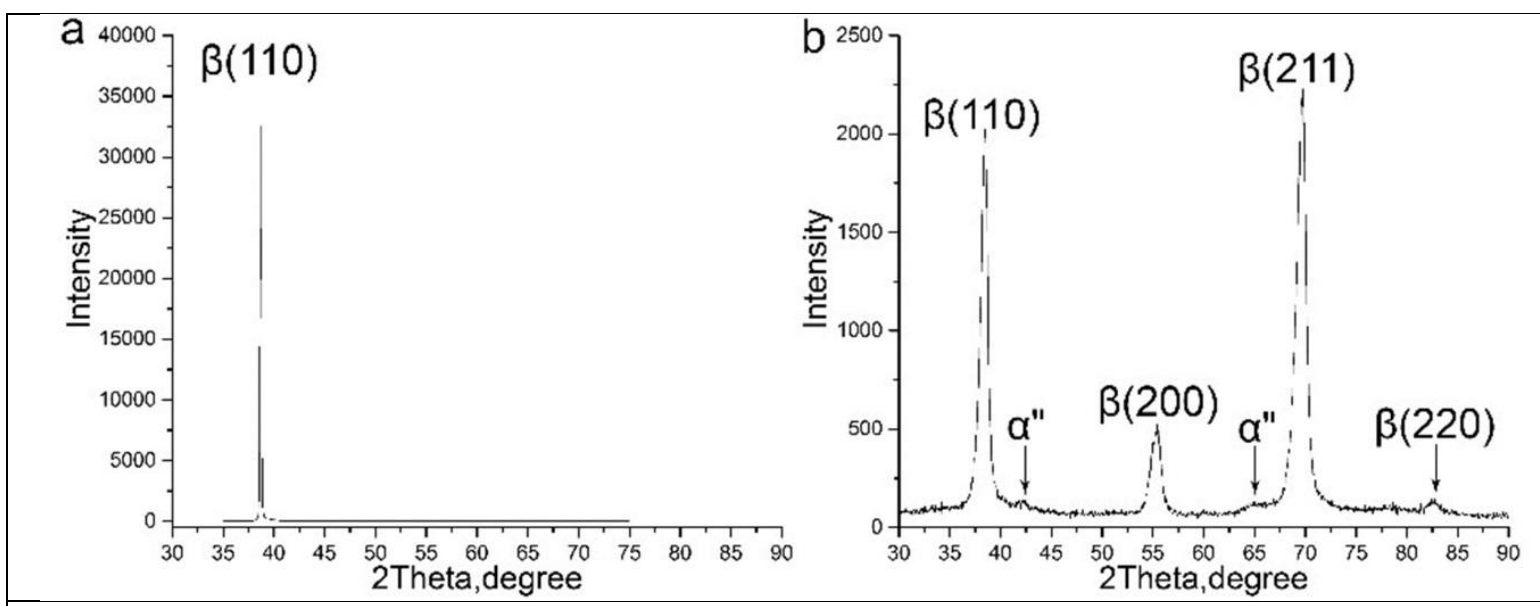

Fig. 9. (a) XRD result of Extruded-GM showing strong texture and only one peak corresponding to the bcc $\beta$-phase; (b) XRD result of ECAP-GM. Evidence of $\alpha$ "martensite can be observed and the texture has been reduced. 


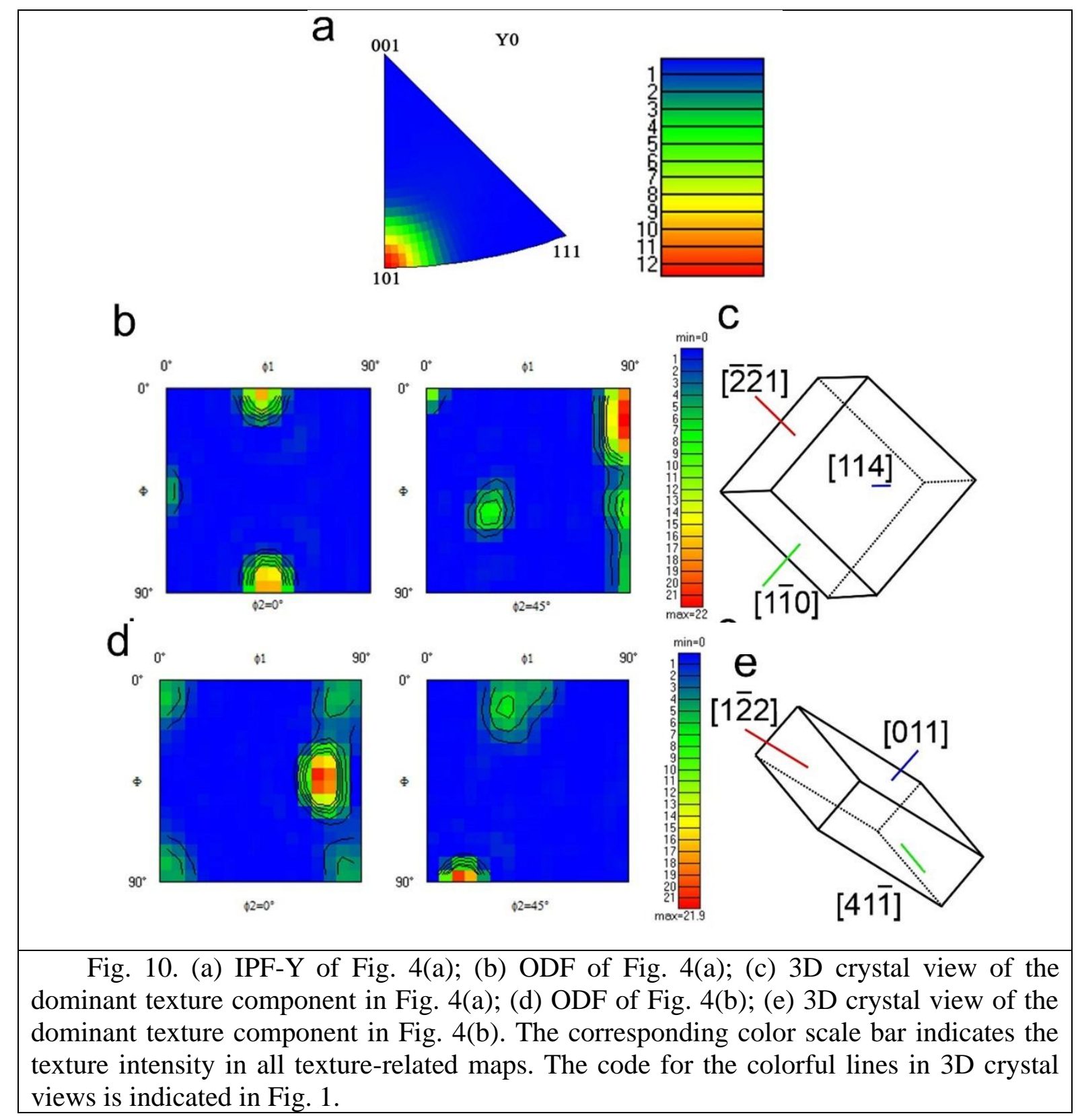




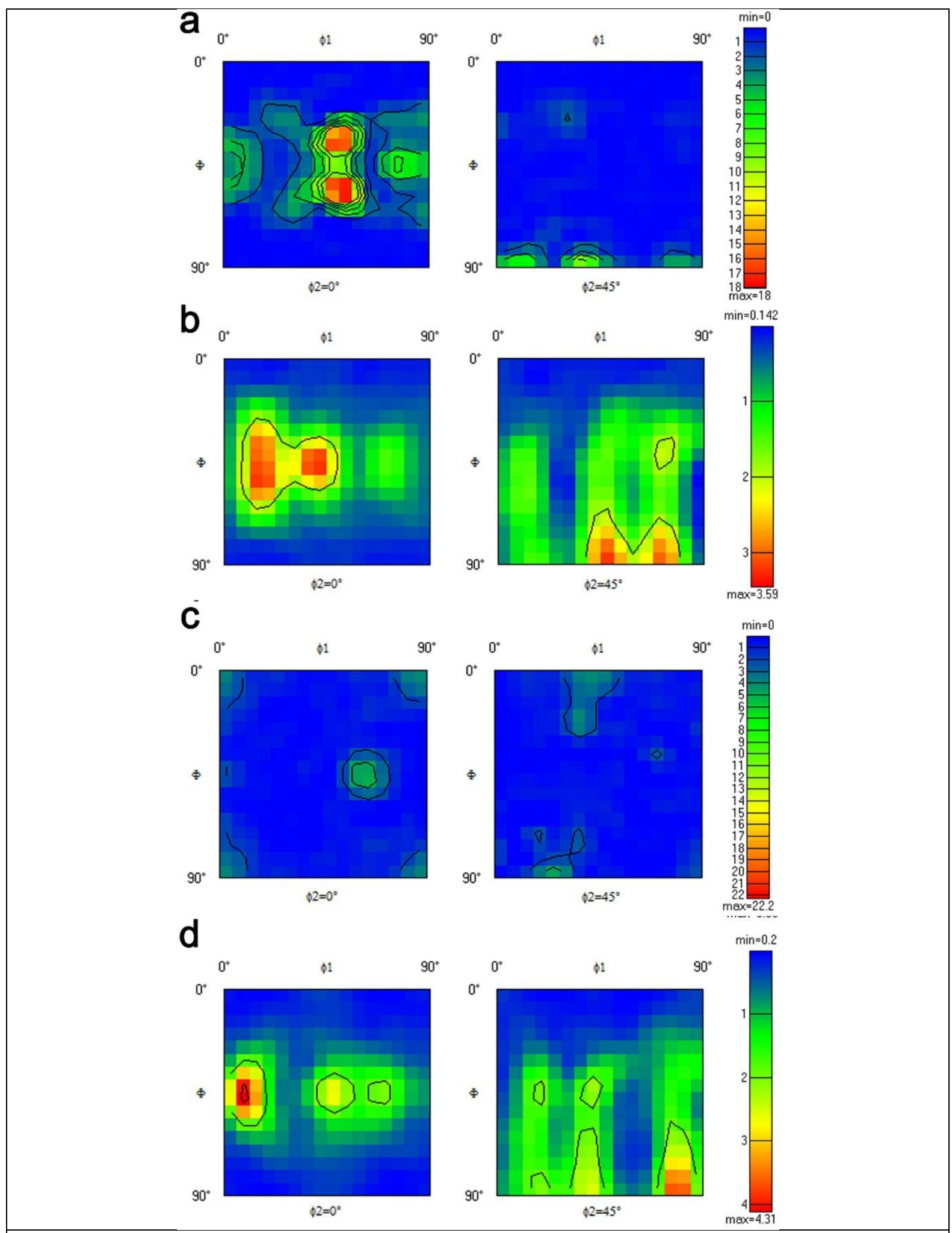

Fig. 11. (a) ODF of matrix Ma in Fig. 8(a); (b) ODF of adiabatic shear band Sa in Fig. 8(a); (c) ODF of matrix Mb in Fig. 8(b); (d) ODF of adiabatic shear band $\mathrm{Sb}$ in Fig. 8(b). 


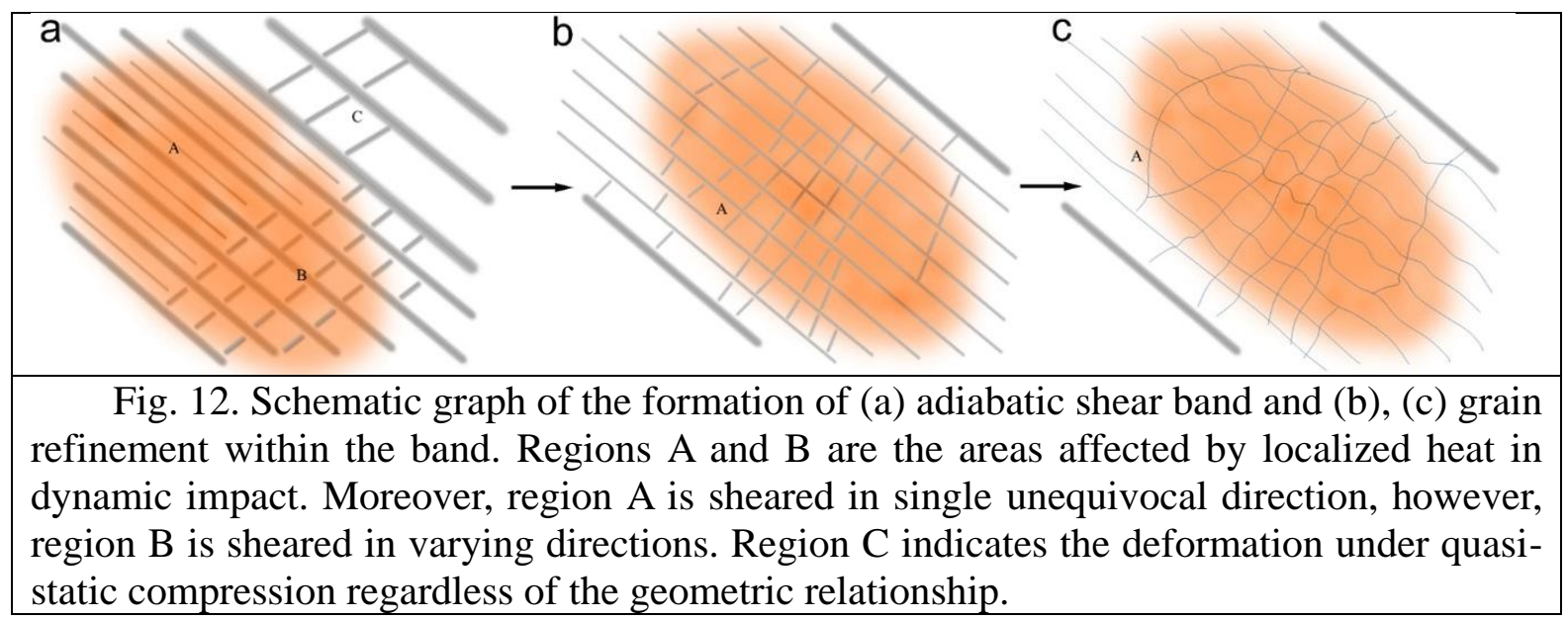




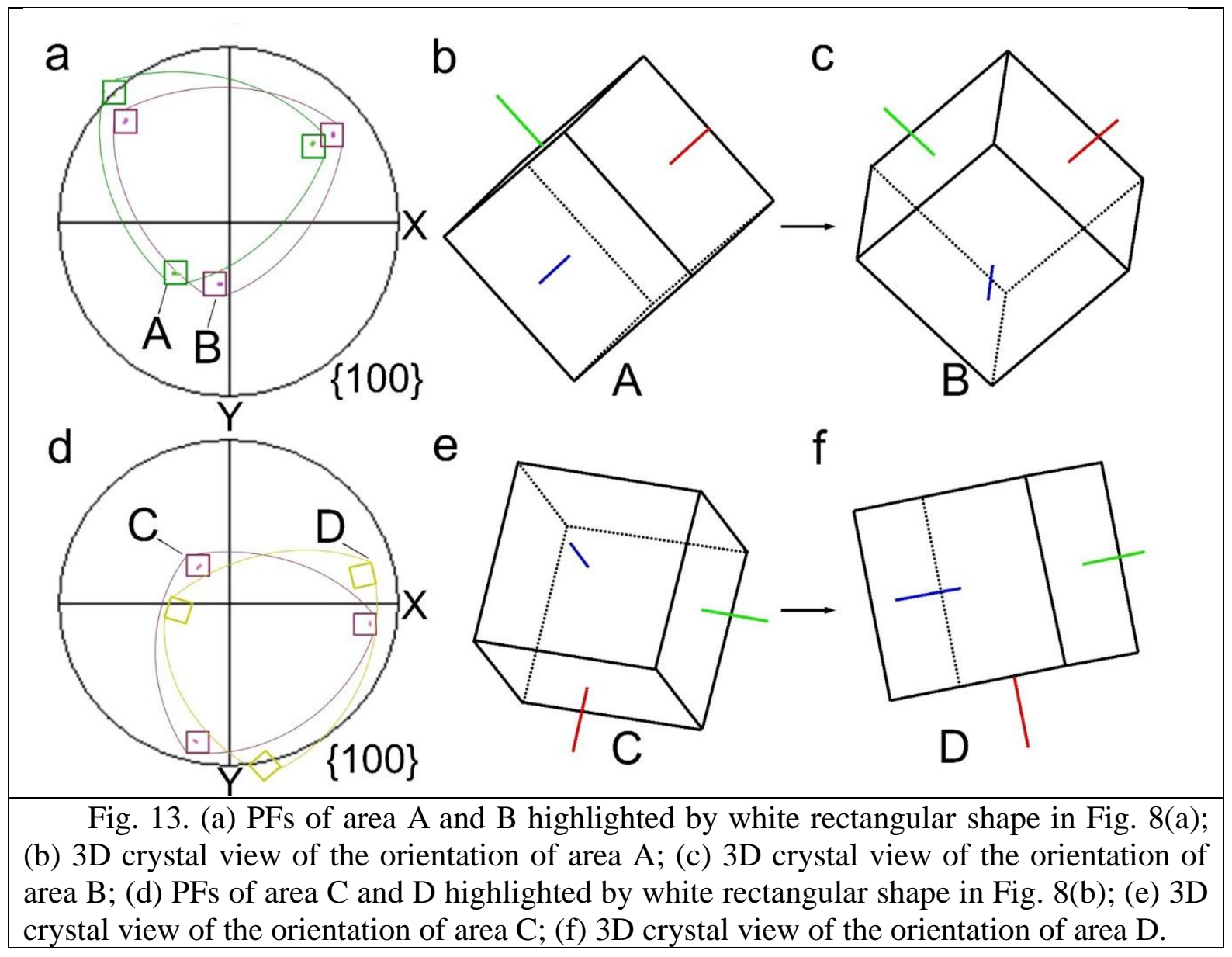


Table 1: The nominal composition of Ti alloy of this work.

\begin{tabular}{|l|l|l|l|l|l|}
\hline Elements & At. \% & Wt. \% & $\begin{array}{l}\text { Density } \\
\text { g/cc }\end{array}$ & Companies & Purities, \% \\
\hline Ti & 73.3 & 58.77 & 4.51 & Alfa & 99.999 \\
\hline $\mathbf{N b}$ & 23 & 35.79 & 8.58 & PMTI & 99.9 \\
\hline Ta & 0.7 & 2.12 & 16.68 & Alfa & 99.95 \\
\hline $\mathbf{Z r}$ & 2 & 3.05 & 6.51 & Alfa & 99.5 \\
\hline $\mathbf{O}$ & 1 & 0.27 & -- & -- & 100.00 \\
\hline Final alloy & 100 & 100 & 5.53 & -- & \\
\hline
\end{tabular}

Table 2: Schmid factors of activated shear directions by compressing in TD and ED of ExtrudedGM.

\begin{tabular}{|c|c|c|c|c|c|c|c|c|c|}
\hline \multirow[b]{2}{*}{ LD } & $\multicolumn{2}{|c|}{110}<110>$ & $\multicolumn{2}{|c|}{110}<111>$ & $\multicolumn{2}{|c|}{112}<111>$ & & & $\{123\}<11$ \\
\hline & $\mathrm{R}^{\mathrm{O}}$ & $\mathrm{m}$ & OR & $\mathrm{m}$ & OR & $\mathrm{m}$ & OR & $\mathrm{m}$ & OR \\
\hline $\begin{array}{l}{[\overline{2} \overline{2} 1]} \\
/ \mathbf{T D}\end{array}$ & one & $\begin{array}{l}\mathrm{N} \\
\text { one }\end{array}$ & $(0 \overline{11})[11$ & $0.41^{-}$ & $(1 \overline{1})[\overline{1}$ & $0.47^{-}$ & $(\overline{3} 2)[11$ & $0.31^{-}$ & $(12 \overline{3})[$ \\
\hline & & & $(10 \overline{1})[\overline{111}]$ & $0.41^{-}$ & & & $(21 \overline{3})[\overline{11}$ & $0.46^{-}$ & $(3 \overline{1} \overline{2})[\overline{1}$ \\
\hline
\end{tabular}




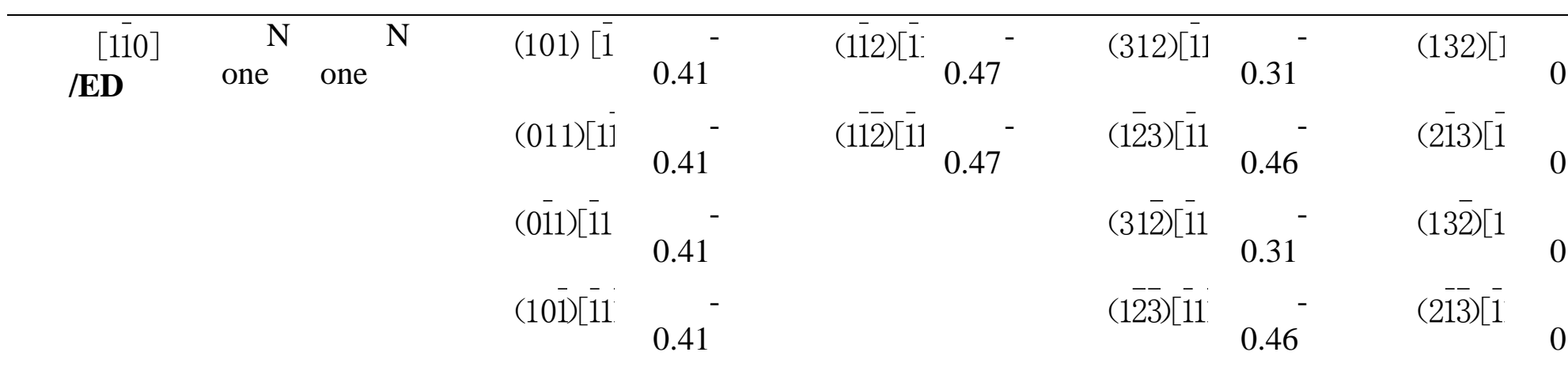

Note: LD represents the loading direction; SD represents potential shear direction; OR is the activated orientation. 
Table 3: Schmid factors of activated shear directions by compressing in TD and ED of ECAPGM.

\begin{tabular}{|c|c|c|c|c|c|c|c|c|c|}
\hline \multirow{2}{*}{$\begin{array}{l}\text { SD } \\
\text { LD }\end{array}$} & $\multicolumn{4}{|c|}{110}<110\rangle$ & $\multicolumn{2}{|c|}{110}<111\rangle$ & $\multicolumn{2}{|c|}{112}<111\rangle$ & \multirow[b]{2}{*}{ OR } \\
\hline & OR & $\mathrm{m}$ & OR & $\mathrm{m}$ & OR & $\mathrm{m}$ & OR & $\mathrm{n}$ & \\
\hline \multirow{3}{*}{$\begin{array}{l}{[1 \overline{2} 2]} \\
/ \mathbf{T D}\end{array}$} & None & $\mathrm{N}$ & None & $\mathrm{N}$ & $(101)[111]$ & - & $(2 \overline{1} 11)[11 \overline{1}]$ & - & (213) \\
\hline & & one & & one & & 0.41 & & 0.47 & \\
\hline & & & & & $(1 \overline{10})[11 \overline{1}]$ & $0.41^{-}$ & & & $(3 \overline{1}$ \\
\hline \multirow[t]{5}{*}{$/^{[41 \overline{1}]}$} & (101) [1] & $0.42^{-}$ & (101) $[10]$ & $0.42^{-}$ & $(110)[111]$ & $0.45^{-}$ & $(211)[111]$ & $0.42^{-}$ & (31 \\
\hline & $(110)[\overline{1}]$ & $0.42^{-}$ & $(\overline{110})\left[\overline{110_{-}^{-}}\right.$ & $0.42^{-}$ & (101)[111] & $0.41^{-}$ & $(12 \overline{1})[\overline{11} 1]$ & $0.37^{-}$ & (32 \\
\hline & (101)[11 & $0.42^{-}$ & $(10 \overline{1})[10$ & $0.42^{-}$ & $(\overline{110})[\overline{111}]$ & $0.41^{-}$ & $(2 \overline{11})[\overline{111}]$ & $0.47^{-}$ & $(23 \overline{1})[$ \\
\hline & $(110)[1]$ & $0.42^{-}$ & $(\overline{110})[110$ & $0.42^{-}$ & $(10 \overline{1})[\overline{111}]$ & $0.45^{-}$ & $(11 \overline{2})[\overline{111}]$ & $0.37^{-}$ & $(13 \overline{2})$ \\
\hline & & & & & & & $(2 \overline{11})[\overline{111}]$ & $0.42^{-}$ & (21 \\
\hline
\end{tabular}

Note: LD represents the loading direction; SD represents potential shear direction; OR is the activated orientation. 
Table 4: Schmid factors of activated shear directions by compressing in crystallographic direction $[3 \overline{3} 2]$.

\begin{tabular}{|c|c|c|c|c|c|c|c|c|}
\hline \multirow{2}{*}{$\mathrm{LD}^{\mathrm{SD}}$} & $\multicolumn{2}{|c|}{110}<110\rangle$ & $\multicolumn{2}{|c|}{110}<111\rangle$ & $\multicolumn{2}{|c|}{112}<111\rangle$ & $\multicolumn{2}{|c|}{123}<111\rangle$ \\
\hline & OR & $\mathrm{m}$ & OR & $\mathrm{m}$ & OR & $\mathrm{n}$ & OR & $\mathrm{n}$ \\
\hline$[\overline{3} 32]$ & None & one & $(101)[\overline{111}]$ & $0.37^{-}$ & $(\overline{112})[\overline{111}]$ & $0.43^{-}$ & $(\overline{12} 3)[\overline{11} 1]$ & $0.42^{-}$ \\
\hline & & & $(\overline{011})[\overline{11} 1]$ & $0.37^{-}$ & & & $(2 \overline{13})[\overline{11} 1]$ & $0.42^{-}$ \\
\hline
\end{tabular}

Note: LD represents the loading direction; SD represents potential shear direction; OR is the activated orientation. 


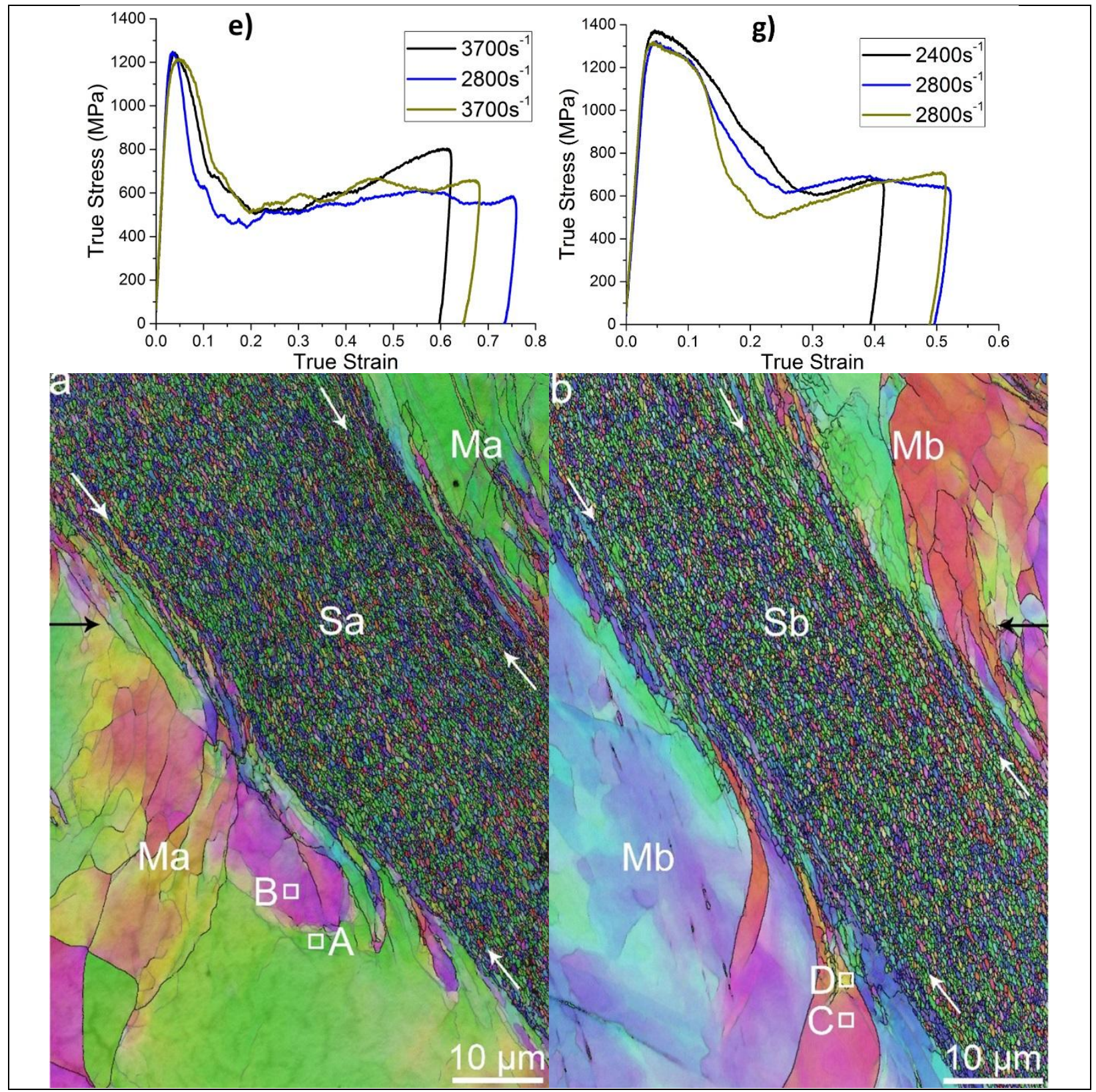

